\title{
Iron(II) and Zinc(II) Complexes with Designed pybox Ligand for the Asymmetric Aqueous Mukaiyama-Aldol Reactions
}

\author{
Joanna Jankowska, Joanna Paradowska, Bartosz Rakiel and Jacek Mlynarski* \\ Institute of Organic Chemistry, Polish Academy of Sciences, Kasprzaka 44/52 \\ 01-224 Warsaw, POLAND \\ mlynar@icho.edu.pl
}

\section{Contents}

Experimental

page $\mathrm{S}-2$

Synthesis of TPS-he-pybox (3c)

page S-2

General Procedure for the Asymmetric Aldol Reactions

page $\mathrm{S}-4$

Characterization of compounds $\mathbf{7 a - k}$

page $\mathrm{S}-4$

References

page $\mathrm{S}-7$

${ }^{1} \mathrm{H}$ NMR spectra of $\mathbf{S 2}$

page S-9

${ }^{13} \mathrm{H}$ NMR spectra of $\mathbf{S 2}$

page $\mathrm{S}-10$

${ }^{1} \mathrm{H}$ NMR spectra of $\mathbf{S 3}$

page S-11

${ }^{13} \mathrm{H}$ NMR spectra of $\mathbf{S 3}$

page S-12

${ }^{1} \mathrm{H}$ NMR spectra of $\mathbf{3 c}$

page S-13

${ }^{13} \mathrm{H}$ NMR spectra of $\mathbf{3 c}$

page $\mathrm{S}-14$

HPLC data of compounds $\mathbf{7 a - 7 k}$ (Table 3)

page $\mathrm{S}-15$

HPLC data of compound 7i (Table 4)

page $\mathrm{S}-23$

HPLC data of compound $\mathbf{7 j}$ (Table 4)

page S-25 


\section{Experimental}

General: Optical rotations were measured with a Digital Polarimeter at room temperature. ${ }^{1} \mathrm{H}$ NMR spectra were recorded on 200 and $400 \mathrm{MHz}$ spectrometers in $\mathrm{CDCl}_{3}$ with $\mathrm{Me}_{4} \mathrm{Si}$ as internal standard. High resolution mass spectra were taken on a mass spectrometer with timeof-flight (TOF) detector. Reactions were controlled using TLC on silica [alu-plates $(0.2 \mathrm{~mm})$ ]. All reagents and solvents were purified and dried according to common methods. All organic solutions were dried over $\mathrm{Na}_{2} \mathrm{SO}_{4}$. Reaction products were purified by flash chromatography using silica gel 60 (240-400 mesh). HPLC analysis were performed on HPLC system equipped with chiral stationary phase columns, detection at $254 \mathrm{~nm}$.

For the aldol reactions promoted by $\mathrm{Fe}^{\mathrm{II}}$ complex all used solvents, while magnetically stirred were deoxygenated by bubbling argon through it for $30 \mathrm{~min}$.

Ligands $\mathbf{1},{ }^{1} \mathbf{2} \mathbf{a},{ }^{2} \mathbf{2} \mathbf{b},{ }^{2} \mathbf{3 a},{ }^{3} \mathbf{3} \mathbf{b}^{3}$ are known compounds.

Synthesis of TPS-he-pybox (3c):

Synthesis was performed using modified procedure of S. Iwasa et al. ${ }^{3}$
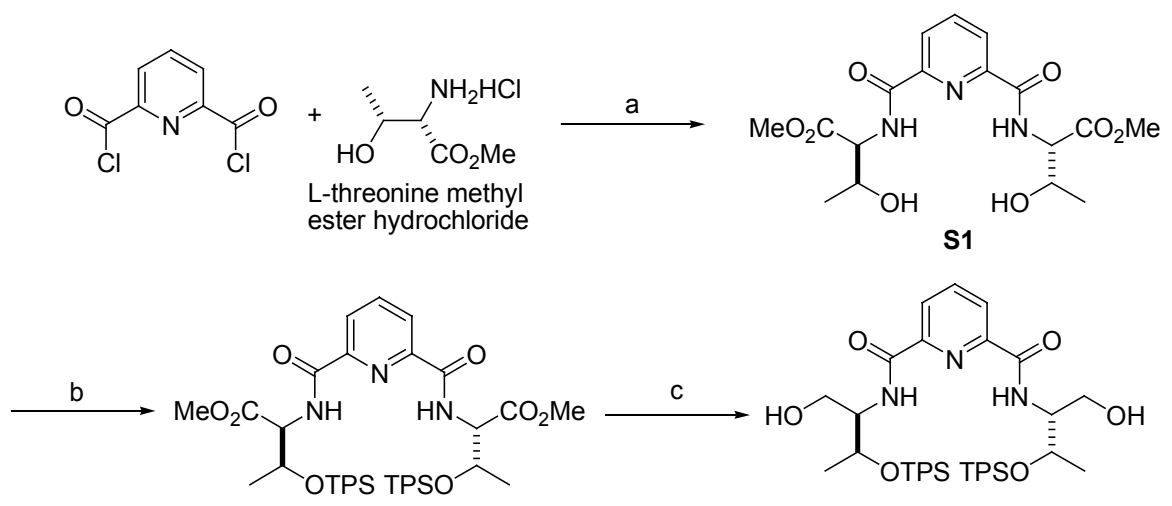

S2

S3

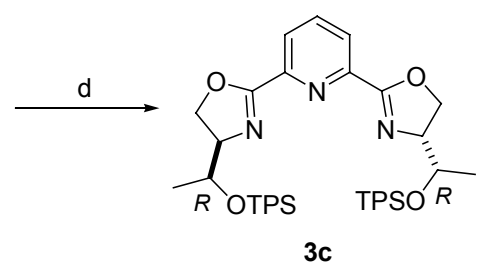

Scheme S-1. Reagents and conditions: a) $\mathrm{Et}_{3} \mathrm{~N}, \mathrm{CHCl}_{3}$, rt, $12 \mathrm{~h}$; b) TPSCl, imidazole, DCM, rt, $12 \mathrm{~h}$; c) $\mathrm{LiBH}_{4}$, THF, $0{ }^{\circ} \mathrm{C}$-rt, $12 \mathrm{~h}$; d) TPP, imidazole, $\mathrm{CCl}_{4}$, DCM, rt, $12 \mathrm{~h}$. 
Preparation of S1: To a solution of L-threonine methyl ester hydrochloride (5.05 g, 29.7 mmol) in chloroform $(45 \mathrm{~mL})$ triethylamine $(10.0 \mathrm{~mL}, 72.1 \mathrm{mmol})$ was added dropwise at 0 ${ }^{\circ} \mathrm{C}$ under Ar. After stirring for $5 \mathrm{~min}$, a solution of 2,6-pyridine dicarbonyldichloride $(3.02 \mathrm{~g}$, $14.8 \mathrm{mmol})$ in chloroform $(25 \mathrm{~mL})$ was added dropwise. When the adding was completed, the reaction mixture was stirred at $\mathrm{rt}$ overnight. Organic phase was washed with water and brine and dried. Chloroform was removed on rotary evaporator and remaining crude ester S1 was submitted to the next step without purification.

Preparation of S2: Solution of ester S1 (5.67 g, $13.4 \mathrm{mmol})$ and imidazole $(2.74 \mathrm{~g}, 40.2$ mmol) in $\mathrm{CH}_{2} \mathrm{Cl}_{2}(50 \mathrm{~mL})$ was treated with tert-butyldiphenylsilyl chloride $(7.67 \mathrm{~g}, 29.5$ mmol). After the reaction mixture was stirred at rt overnight, the solution was evaporated under reduced pressure. Purification by silica gel chromatography (hexane-ethyl acetate: 2:1) gave $11.35 \mathrm{~g}(88 \%)$ of ester S2 as a colourless oil: ${ }^{1} \mathrm{H}$ NMR $(200 \mathrm{MHz}) \delta: 1.04(\mathrm{~s}, 18 \mathrm{H}), 1.07$ (d, 6H, J 6.4 Hz), $3.64(\mathrm{~s}, 6 \mathrm{H}), 4.45-4.56(\mathrm{~m}, 2 \mathrm{H}), 4.86(\mathrm{~d}, 2 \mathrm{H}, J 3.2,9.0 \mathrm{~Hz}), 7.19-7.45$ (m, $12 \mathrm{H}), 7.63-7.70(\mathrm{~m}, 8 \mathrm{H}), 8.05(\mathrm{dd}, 1 \mathrm{H}, J 7.4,8.2 \mathrm{~Hz}), 8.35$ (d, 2H, J 8.0 Hz), $8.42(\mathrm{br} \mathrm{d}, 2 \mathrm{H}, J$ $8.8 \mathrm{~Hz}) ;{ }^{13} \mathrm{C}$ NMR $(50 \mathrm{MHz}) \delta: 19.2,20.1,52.2,58.1,70.4,125.5,127.4,127.6,129.6,129.8$, 133.1, 133.9, 135.8, 138.8, 148.9, 163.6, 170.3; IR (film): 3411, 2953, 2932, 2858, 1750, 1684, 1517, $1112 \mathrm{~cm}^{-1}$; MS-HR: calculated for $\mathrm{C}_{49} \mathrm{H}_{59} \mathrm{~N}_{3} \mathrm{O}_{8} \mathrm{Si}_{2},[M+\mathrm{Na}]^{+}$: 896.3733; found: 896.3727.

Preparation of S3: To a suspension of lithium borohydride $(0.86 \mathrm{~g}, 39.0 \mathrm{mmol})$ in dry THF $(50 \mathrm{~mL})$ a solution of the $\mathbf{S 2}(11.35 \mathrm{~g}, 13.0 \mathrm{mmol})$ in THF $(50 \mathrm{~mL})$ was added dropwise over $20 \mathrm{~min}$ at $0{ }^{\circ} \mathrm{C}$. The reaction mixture was allowed to warm to $\mathrm{rt}$ and stirred overnight. After the reaction was finished $10 \mathrm{~mL}$ of water was carefully added and reaction mixture was extracted with dichloromethane. The combined organic layers were washed with brine, dried and concentrated. Purification by silica gel chromatography (hexane-ethyl acetate: 2:3) gave $4.50 \mathrm{~g}(42 \%)$ of diol $\mathbf{S 3}$ as a colourless oil: ${ }^{1} \mathrm{H}$ NMR $(200 \mathrm{MHz}) \delta: 1.04(\mathrm{~s}, 18 \mathrm{H}), 1.05(\mathrm{~d}, 6 \mathrm{H}$, $J$ 6.4 Hz), 2.25-2.45 (br s, 2H, OH), 3.67-3.84 (m, 4H), 4.00-4.13 (m, 2H), 4.25-4.36 (m, 2 H), 7.28-7.44 (m, 12H), 7.60-7.70 (m, 8H), 7.99 (d, 1H, J 8.6 Hz), 8.10 (dd, 1H, J 7.4, 8.2 $\mathrm{Hz}), 8.35$ (d, 2H, J 8.0 Hz); ${ }^{13} \mathrm{C}$ NMR (50 MHz) $\delta: 19.3,20.4,27.1,57.1,62.9,69.3,125.3$, 127.6, 127.8, 129.8, 129.9, 132.9, 133.9, 135.7, 138.9, 149.0, 164.2; IR (film): 3408, 3071, 2958, 2932, 2858, 1670, 1525, $1111 \mathrm{~cm}^{-1}$; MS-HR: calculated for $\mathrm{C}_{47} \mathrm{H}_{59} \mathrm{~N}_{3} \mathrm{O}_{6} \mathrm{Si}_{2},[M+\mathrm{Na}]^{+}$: 840.3835; found: 840.38037 .

Preparation of TPS-he-pybox 3c: Diol S3 (4.24 g, $5.2 \mathrm{mmol})$, imidazole (3.53 g, $36.4 \mathrm{mmol})$ and triphenyphosphine $(4.27 \mathrm{~g}, 16.3 \mathrm{mmol})$ were disolved in dichloromethane $(30 \mathrm{~mL})$ and stirred at $\mathrm{rt}$ under Ar. To the resulting solution carbon tetrachloride $(8.5 \mathrm{~mL})$ was added and the mixture was stirred at $\mathrm{rt}$ overnight. All volatiles were then removed under reduced pressure. Purification by silica gel chromatography (hexane-ethyl acetate: $3: 2$ ) gave $1.80 \mathrm{~g}$ $(45 \%)$ of pybox $3 \mathrm{c}$ as a viscous oil: $[\alpha]_{\mathrm{D}}-45.1\left(c 1.05\right.$ in $\left.\mathrm{CHCl}_{3}\right) ;{ }^{1} \mathrm{H}$ NMR $(400 \mathrm{MHz}) \delta$ : 1.02 (d, 6H, J 6.4 Hz), 1.04 (s, 18H), 4.18-4.32 (m, 2H), 4.40-4.50 (m, 4H), 4.60-4.70 (m, 2H), 7.34-7.39 (m, 8H), 7.40-7.45 (m, 4H), 7.65-7.69 (m, 8H), 7.81 (dd, 1H, J 7.7, 8.1 Hz), $8.10(\mathrm{~d}, 2 \mathrm{H}, J 7.8 \mathrm{~Hz}) ;{ }^{13} \mathrm{C}$ NMR $(100 \mathrm{MHz}) \delta: 17.5,19.2,26.9,69.0,69.7,71.4,125.7$, 127.5, 127.7, 129.6, 129.7, 133.8, 134.1, 135.7, 135.8, 137.1, 146.8, 163.2; IR (film): 3382, 3223, 3072, 2690, 2932, 2894, 2858, 2252, 1675, 1514, 1427, $1111 \mathrm{~cm}^{-1}$; MS-HR: calculated for $\mathrm{C}_{47} \mathrm{H}_{55} \mathrm{~N}_{3} \mathrm{O}_{4} \mathrm{Si}_{2},[M+\mathrm{H}]^{+}$: 782.3804; found: 782.3809 . 
General Procedure for the Asymmetric Aldol Reactions Catalyzed by Fe $\mathrm{F}^{\mathrm{II}}$ Salt: Mixture of TPS-he-pybox (3c) (47 mg, $0.06 \mathrm{mmol}, 12 \mathrm{~mol} \%$ ) and iron(II) chloride (6.5 mg, 0.05 mmol, $10 \mathrm{~mol} \%$ ) in deoxygenated $\mathrm{EtOH} / \mathrm{H}_{2} \mathrm{O}(9 / 1) 1.5 \mathrm{~mL}$ was stirred at $0{ }^{\circ} \mathrm{C}$ under Ar until all the solid was observed to dissolve (15-20 min.). To the resulting deep-red solution silyl enol ether $5(230 \mu \mathrm{L}, 1.0 \mathrm{mmol}, 2$ equivalents $)$ and appropriate aldehyde $(0.5 \mathrm{mmol})$ were added and resulting solution was stirred at $0{ }^{\circ} \mathrm{C}$ for $5 \mathrm{~h}$ under Ar. The reaction was diluted with MTBE and washed with water and brine. Organic phase was dried, evaporated to dryness and the residue was purified by silica gel chromatography (typically, AcOEt/hexane, $1: 4)$.

General Procedure for the Asymmetric Aldol Reactions Catalyzed by $\mathrm{Zn}^{\text {II }}$ Salt: Mixture of TPS-he-pybox (3c) (23 mg, $0.03 \mathrm{mmol}, 12 \mathrm{~mol} \%)$ and zinc(II) trifluoromethanesulfonate (9 mg, $0.025 \mathrm{mmol}, 10 \mathrm{~mol} \%$ ) in $\mathrm{EtOH} / \mathrm{H}_{2} \mathrm{O}(9 / 1) 1.0 \mathrm{~mL}$ was stirred at $-20{ }^{\circ} \mathrm{C}(15-20 \mathrm{~min}$.). To the resulting homogeneous solution silyl enol ether 5 (115 $\mu \mathrm{L}, 0.5 \mathrm{mmol}, 2$ equivalents) and appropriate aldehyde $(0.25 \mathrm{mmol})$ were added and resulting homogeneous solution was left in refrigerator at $-20{ }^{\circ} \mathrm{C}$ overnight without stirring. The reaction mixture was poured onto silica gel column and eluted by AcOEt/hexane (1:4) to yield desired aldols.

(2S,3S)-3-Hydroxy-2-methyl-1,3-diphenyl-propan-1-one (7a) ${ }^{4,5,6}$

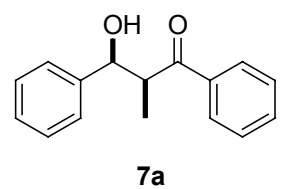

${ }^{1} \mathrm{H}$ NMR $(200 \mathrm{MHz}) \delta(s y n): 1.19$ (d, 3H, J 7.2 Hz), 3.67 (d, 1H, OH, J 1.7 Hz), 4.11 (dq, 1H, $J 3.0,7.3 \mathrm{~Hz}$ ), 5.23 (brs, $1 \mathrm{H}), 7.20-7.65$ (m, 8H), 7.85-8.20 (m, 2H).

${ }^{13} \mathrm{C}$ NMR (50 MHz) $\delta: 11.1,47.1,73.0,125.9,127.3,128.2,128.4,128.7,133.5,135.6$, $141.7,205.8$.

$[\alpha]_{\mathrm{D}}+3.5\left(c 0.1\right.$ in $\mathrm{CHCl}_{3}, 85 \%$ ee), lit.[4] for $\operatorname{syn}-(2 S, 3 S)-5 \mathrm{a}:[\alpha]_{\mathrm{D}}+11.7$ (c 1.03 in $\mathrm{CHCl}_{3}$, $95 \%$ ee);

HPLC (Daicel Chiralpak AD-H, hexane $/ i-\mathrm{PrOH}=9 / 1$, flow rate $=1 \mathrm{~mL} / \mathrm{min}$; syn isomer: $\mathrm{t}_{1}=$ 9.9 min, $\mathrm{t}_{2}=12.2 \mathrm{~min}$ (major), anti isomer: $\mathrm{t}_{1}=14.6 \mathrm{~min}, \mathrm{t}_{2}=16.0 \mathrm{~min}$.

\section{3-Hydroxy-3-(4-methoxy-phenyl)-2-methyl-1-phenyl-propan-1-one (7b) $)^{5,6,7}$}

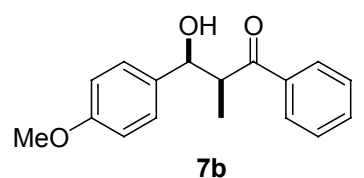

${ }^{1} \mathrm{H}$ NMR $(200 \mathrm{MHz}) \delta(s y n): 1.27(\mathrm{~d}, 3 \mathrm{H}, J 7.2 \mathrm{~Hz}), 3.57$ (d, 1H, $\left.J 2.0 \mathrm{~Hz}\right), 3.67(\mathrm{dq}, 1 \mathrm{H}, J$ 3.6, 7.1 Hz), 3.78 (s, 3H), 5.16-5.20 (m, 1H), 6.80-6.84 (m, 2H), 7.24-7.38 (m, 2H), 7.40-7.64 $(\mathrm{m}, 3 \mathrm{H}), 7.84-8.20(\mathrm{~m}, 2 \mathrm{H})$.

${ }^{13} \mathrm{C}$ NMR $(50 \mathrm{MHz}) \delta: 11.5,47.2,55.2,72.9,113.6,127.1,127.8,128.4,128.7,133.4,133.9$, 135.7, 205.6.

HPLC (Daicel Chiralpak AD-H, hexane $/ i-\mathrm{PrOH}=9 / 1$, flow rate $=1 \mathrm{~mL} / \mathrm{min}$; syn isomer: $\mathrm{t}_{1}=$ 15.9 min, $\mathrm{t}_{2}=17.9 \mathrm{~min}$ (major), anti isomer: $\mathrm{t}_{1}=25.0 \mathrm{~min}, \mathrm{t}_{2}=25.8 \mathrm{~min}$. 
Hydroxy-3-(2-methoxy-phenyl)-2-methyl-1-phenyl-propan-1-one (7c) $)^{5,6,8}$

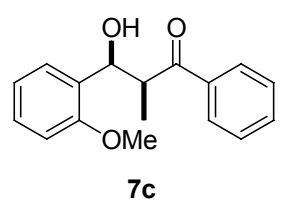

${ }^{1} \mathrm{H}$ NMR $(200 \mathrm{MHz}) \delta(s y n): 1.15(\mathrm{~d}, 3 \mathrm{H}, J 7.2 \mathrm{~Hz}), 3.56(\mathrm{~d}, 1 \mathrm{H}, \mathrm{OH}, J 2.7 \mathrm{~Hz}), 3.58(\mathrm{~s}, 3 \mathrm{H})$, $3.94(\mathrm{dq}, 1 \mathrm{H}, J 3.0,7.1 \mathrm{~Hz}), 5.41(\mathrm{brs}, 1 \mathrm{H}), 6.85$ (dd, 1H, $J 1.0,8.2 \mathrm{~Hz}), 6.92-7.20(\mathrm{~m}, 1 \mathrm{H})$, 7.18-7.30 (m, 1H), 7.40-7.63 (m, 4H), 7.94-8.04 (m, 2H).

${ }^{13} \mathrm{C}$ NMR $(50 \mathrm{MHz}) \delta: 10.8,44.1,55.1,69.2,109.9,120.5,127.6,128.1,128.4,128.5,129.5$, 133.2, 135.9, 155.4, 205.7.

HPLC (Daicel Chiralpak AD-H, hexane $/ i-\mathrm{PrOH}=9 / 1$, flow rate $=1 \mathrm{~mL} / \mathrm{min}$; syn isomer: $\mathrm{t}_{1}=$ 12.5 min, $\mathrm{t}_{2}=18.9$ min (major), anti isomer: $\mathrm{t}_{1}=23.6 \mathrm{~min}, \mathrm{t}_{2}=23.8 \mathrm{~min}$.

3-Hydroxy-3-(4-methyl-phenyl)-2-methyl-1-phenyl-propan-1-one (7d)

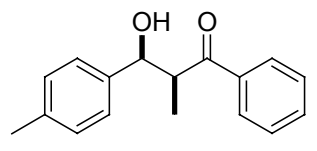

$7 d$

${ }^{1} \mathrm{H}$ NMR $(200 \mathrm{MHz}) \delta(s y n): 1.19(\mathrm{~d}, 3 \mathrm{H}, J 7.2 \mathrm{~Hz}), 2.32(\mathrm{~s}, 3 \mathrm{H}), 3.60(\mathrm{~d}, 1 \mathrm{H}, \mathrm{OH}, J 1.7 \mathrm{~Hz})$, $3.68(\mathrm{dq}, 1 \mathrm{H}, J 3.3,7.1 \mathrm{~Hz}), 5.19(\mathrm{~d}, 1 \mathrm{H}, J 0.8 \mathrm{~Hz}), 7.08-8.00(\mathrm{~m}, 9 \mathrm{H})$.

${ }^{13} \mathrm{C}$ NMR (50 MHz) $\delta: 11.3,21.0,47.1,73.0,125.9,128.4,128.7,128.8,133.4,135.6,136.8$, $138.8,205.5$.

HPLC (Daicel Chiralpak AD-H, hexane $/ i-\mathrm{PrOH}=9 / 1$, flow rate $=1 \mathrm{~mL} / \mathrm{min}$; syn isomer: $\mathrm{t}_{1}=$ $10.7 \mathrm{~min}, \mathrm{t}_{2}=12.2 \mathrm{~min}$ (major), anti isomer: $\mathrm{t}_{1}=16.6 \mathrm{~min}, \mathrm{t}_{2}=17.8 \mathrm{~min}$.

3-(2-Chlorophenyl)-3-hydroxy-2-methyl-1-phenyl-propan-1-one (7e) ${ }^{6}$

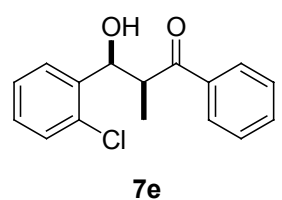

${ }^{1} \mathrm{H}$ NMR $(200 \mathrm{MHz}) \delta(s y n): 1.10$ (d, 3H, J 7.2 Hz), 3.94 (dq, 1H, J 5.5, 7.4 Hz), 4.03 (d, 1H, $\mathrm{OH}, J 1.9 \mathrm{~Hz}), 5.55$ (brs, $1 \mathrm{H}), 7.20-7.72(\mathrm{~m}, 7 \mathrm{H}), 7.98-8.03(\mathrm{~m}, 2 \mathrm{H})$.

${ }^{13} \mathrm{C}$ NMR $(50 \mathrm{MHz}) \delta: 10.4,42.9,69.7,126.7,128.4,128.6,128.8,129.2,131.0,133.7$, 135.5, 138.6, 206.3.

HPLC (Daicel Chiralpak AD-H, hexane $/ i-\operatorname{PrOH}=9 / 1$, flow rate $=1 \mathrm{~mL} / \mathrm{min}$; syn isomer: $\mathrm{t}_{1}=$ 7.9 min, $\mathrm{t}_{2}=11.0 \mathrm{~min}$ (major), anti isomer: $\mathrm{t}_{1}=14.0 \mathrm{~min}, \mathrm{t}_{2}=16.6 \mathrm{~min}$. 


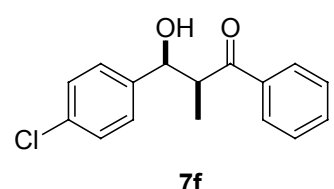

${ }^{1} \mathrm{H}$ NMR (200 MHz) $\delta(s y n): 1.17(\mathrm{~d}, 3 \mathrm{H}, J 7.2 \mathrm{~Hz}), 3.64(\mathrm{dq}, 1 \mathrm{H}, J 3.2,7.3 \mathrm{~Hz}), 3.82(\mathrm{~d}, 1 \mathrm{H}$, $J 1.9 \mathrm{~Hz}), 5.20$ (t, $1 \mathrm{H}, J 3.0 \mathrm{~Hz}), 7.25-7.65$ (m, 8H), 7.88-8.10 (m, 2H).

${ }^{13} \mathrm{C}$ NMR $(50 \mathrm{MHz}) \delta: 11.1,46.8,72.4,127.4,127.9,128.6,128.8,132.9,133.6,135.4$, 140.3, 205.4.

HPLC (Daicel Chiralpak AD-H, hexane $/ i-\operatorname{PrOH}=9 / 1$, flow rate $=1 \mathrm{~mL} / \mathrm{min}$; syn isomer: $\mathrm{t}_{1}=$ 11.1 min, $\mathrm{t}_{2}=12.5 \mathrm{~min}$ (major), anti isomer: $\mathrm{t}_{1}=15.7 \mathrm{~min}$ (major), $\mathrm{t}_{2}=20.6 \mathrm{~min}$.

\section{3-Hydroxy-3-(4-nitro)-2-methyl-1-phenyl-propan-1-one (7g) ${ }^{9,11}$}

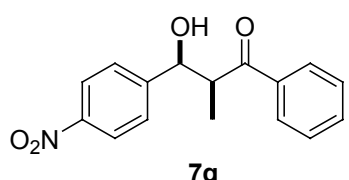

${ }^{1} \mathrm{H}$ NMR (200 MHz) $\delta(s y n): 1.20(\mathrm{~d}, 3 \mathrm{H}, J 7.2 \mathrm{~Hz}), 3.55$ (d, 1H, $\left.J 5.7 \mathrm{~Hz}\right), 3.70(\mathrm{dq}, 1 \mathrm{H}, J$ 2.7, $7.3 \mathrm{~Hz}$ ), 4.05 (d, 1H, J $1.9 \mathrm{~Hz}, \mathrm{OH}), 5.36$ (br s, 1H), 7.47-7.63 (m, 3H), 7.92-7.98 (m, $2 \mathrm{H}), 8.20-8.25(\mathrm{~m}, 2 \mathrm{H})$.

${ }^{13} \mathrm{C}$ NMR $(50 \mathrm{MHz}) \delta: 10.9,46.4,72.3,123.4,126.9,128.5,128.9,133.7,133.9,135.1$, 149.1, 205.2.

HPLC (Daicel Chiralpak AD-H, hexane $/ i-\mathrm{PrOH}=9 / 1$, flow rate $=1 \mathrm{~mL} / \mathrm{min}$; syn isomer: $\mathrm{t}_{1}=$ $19.2 \mathrm{~min}$ (major), $\mathrm{t}_{2}=20.9 \mathrm{~min}$, anti isomer: $\mathrm{t}_{1}=23.3 \mathrm{~min}, \mathrm{t}_{2}=45.3 \mathrm{~min}$.

\section{3-Hydroxy-3-pirydyno-2-methyl-1-phenyl-propan-1-one (7h) ${ }^{5}$}

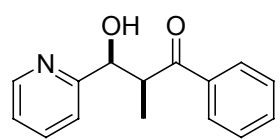

$7 \mathrm{~h}$

${ }^{1} \mathrm{H}$ NMR (200 MHz) $\delta(s y n): 1.15$ (d, 3H, J 7.4 Hz), $4.08(\mathrm{~d}, 1 \mathrm{H}, J 4.1 \mathrm{~Hz}), 4.20$ (br s, $1 \mathrm{H}$, $\mathrm{OH}), 5.22(\mathrm{~d}, 1 \mathrm{H}, J 3.6 \mathrm{~Hz}), 7.18(\mathrm{dq}, 1 \mathrm{H}, J 1.1,4.9 \mathrm{~Hz}), 7.36-7.70(\mathrm{~m}, 6 \mathrm{H}), 7.92-7.97$ (m, $2 \mathrm{H}), 8.50-8.60(\mathrm{~m}, 1 \mathrm{H})$.

${ }^{13} \mathrm{C}$ NMR $(50 \mathrm{MHz}) \delta: 11.6,46.2,73.6,121.2,122.2,128.5,128.6,133.3,136.5,148.7$, 160.9, 205.1.

HPLC (Daicel Chiralpak AS-H, hexane $/ i-\mathrm{PrOH}=9 / 1$, flow rate $=1 \mathrm{~mL} / \mathrm{min}$; syn isomer: $\mathrm{t}_{1}=$ 8.4 min, $\mathrm{t}_{2}=11.7 \mathrm{~min}$, anti isomer: $\mathrm{t}_{1}=12.7, \mathrm{t}_{2}=14.9 \mathrm{~min}$. 


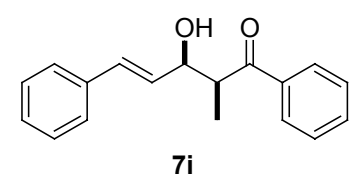

${ }^{1} \mathrm{H}$ NMR (200 MHz) $\delta(s y n): 1.36$ (d, 3H, J $\left.7.4 \mathrm{~Hz}\right), 3.40$ (brs, 1H, OH), 3.64 (dq, 1H, J 3.5, $7.3 \mathrm{~Hz}), 4.72-4.81(\mathrm{~m}, 1 \mathrm{H}), 6.24(\mathrm{dd}, 1 \mathrm{H}, J 5.7,16.0 \mathrm{~Hz}), 6.71$ (dd, 1H, J 1.4, $16.0 \mathrm{~Hz}), 7.10-$ $7.70(\mathrm{~m}, 8 \mathrm{H}), 7.80-8.10(\mathrm{~m}, 2 \mathrm{H})$.

${ }^{13} \mathrm{C}$ NMR (50 MHz) $\delta: 11.9,45.7,72.2,126.4,127.5,128.4,128.8,128.7,129.1,130.9$, 133.4, 135.8, 136.6, 205.0.

HPLC (Daicel Chiralpak AD-H, hexane $/ i-\mathrm{PrOH}=9 / 1$, flow rate $=1 \mathrm{~mL} / \mathrm{min}$; syn isomer: $\mathrm{t}_{1}=$ $17.8 \mathrm{~min}, \mathrm{t}_{2}=21.8 \mathrm{~min}$ (major), anti isomer: $\mathrm{t}_{1}=20.0 \mathrm{~min}, \mathrm{t}_{2}=26.9 \mathrm{~min}$.

3-Hydroxy-2-methyl-1,5-diphenyl-pentan-1-one $(\mathbf{7 j})^{5,9,12}$

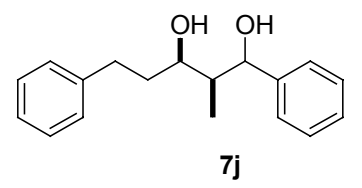

${ }^{1} \mathrm{H}$ NMR $(200 \mathrm{MHz}) \delta(s y n): 1.27(\mathrm{~d}, 3 \mathrm{H}, J 7.2 \mathrm{~Hz}), 1.62-2.04(\mathrm{~m}, 2 \mathrm{H}), 2.62-2.79(\mathrm{~m}, 1 \mathrm{H})$, 2.82-2.98 (m, 1H), $3.16(\mathrm{~d}, 1 \mathrm{H}, \mathrm{OH}, J 1.6 \mathrm{~Hz}), 3.44(\mathrm{dq}, 1 \mathrm{H}, J$ 7.2, $2.8 \mathrm{~Hz}), 4.08(\mathrm{~m}, 1 \mathrm{H})$, 7.12-7.34 (m, 5H), 7.41-7.52 (m, 2H), 7.50-7.63 (m, 3H), 7.86-7.97 (m, 2H).

${ }^{13} \mathrm{C}$ NMR $(50 \mathrm{MHz}) \delta: 11.1,32.3,36.1,44.6,70.6,125.8,128.3,128.4(2 \mathrm{C}), 128.5,128.7$, 133.4, 141.9, 205.7;

HPLC (Daicel Chiralpak AS-H, hexane $/ i-\mathrm{PrOH}=97 / 3$, flow rate $=1 \mathrm{~mL} / \mathrm{min}$; syn isomer: $\mathrm{t}_{1}=$ 12.0 min, $\mathrm{t}_{2}=18.8 \mathrm{~min}$ (major), anti isomer: $\mathrm{t}_{1}=13.4 \mathrm{~min}$ (major), $\mathrm{t}_{2}=29.0 \mathrm{~min}$.

\section{3-Hydroxy-3-(2-methoxy-phenyl)-1-phenyl-propan-1-one (7k) ${ }^{6}$}

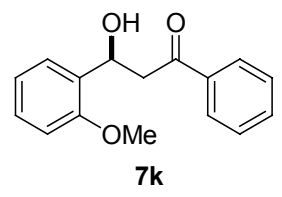

${ }^{1} \mathrm{H}$ NMR (200 MHz) $\delta: 3.24(\mathrm{dd}, 1 \mathrm{H}, J$ 9.0, $17.0 \mathrm{~Hz}), 3.50(\mathrm{dd}, 1 \mathrm{H}, J$ 4.0, $17.0 \mathrm{~Hz}), 3.68(\mathrm{~d}$, $1 \mathrm{H}, \mathrm{OH}, J 4.0 \mathrm{~Hz}), 3.83(\mathrm{~s}, 3 \mathrm{H}), 5.60(\mathrm{~d}, 1 \mathrm{H}, J 8.0 \mathrm{~Hz}), 6.88(\mathrm{~d}, 2 \mathrm{H}, J 8.0 \mathrm{~Hz}), 7.00(\mathrm{td}, 1 \mathrm{H}, J$ 2.0, 7.4 Hz), 7.27 (td, 1H, J 2.0, 7.4 Hz), 7.38-7.62 (m, 4H), 7.90-8.02 (m, 2H).

${ }^{13} \mathrm{C}$ NMR $(50 \mathrm{MHz}) \delta: 45.7,55.2,65.5,110.1,120.8,126.4,128.1,128.3,128.5,131.0$, 133.3, 136.7, 155.6, 200.5.

HPLC (Daicel Chiralpak AS-H, hexane $/ i-\mathrm{PrOH}=9 / 1$, flow rate $=1 \mathrm{~mL} / \mathrm{min}: \mathrm{t}_{1}=14.1 \mathrm{~min}, \mathrm{t}_{2}$ $=19.7$ min (major).

\section{References:}

1. Commercial ligand (Fluka).

2. (a) Iwasa, S.; Nakamura, H.; Nishiyama, H. Heterocycles 2000, 52, 939; (b) Iwasa, S.; Tsushima, S.; Shimada, T.; Nishiyama, H. Tetrahedron 2002, 58, 227. 
3. Iwasa, S.; Tsushima, S.; Nishiyama, K.; Tsuchiya, Y.; Takezawa, F.; Nishiyama, H. Tetrahedron: Asymmetry 2003, 14, 855.

4. Denmark, S.; Wong, K.-T.; Stavenger, R.A. J. Am. Chem. Soc. 1997, 119, 2333.

5. Hamada, T.; Manabe, K.; Ishikawa, S.; Nagayama, S.; Shiro, M.; Kobayashi, S. J. Am. Chem. Soc. 2003, 125, 2989.

6. Jankowska, J.; Mlynarski, J. J. Org. Chem. 2006, 71, 1317.

7. Iwasawa, N.; Mukaiyama, T. Chem. Lett. 1982, 1441.

8. Harrison, C.R. Tetrahedron Lett. 1987, 28, 4135.

9. Shibata, I.; Suwa, T.; Sakakibara, H.; Baba, A. Org. Lett. 2002, 4, 301.

10. Kobayashi, S.; Nagayama, S.; Busujima, T. Tetrahedron 1999, 55, 8739.

11. Shibata, I.; Yamaguchi, T.; Baba, A.; Matsuda, H. Chem. Lett. 1993, 97.

12. Hirama, M.; Garvey, D.S.; Lu, L.D.L.; Masumene, S. Tetrahedron Lett. 1979, 41, 3937. 


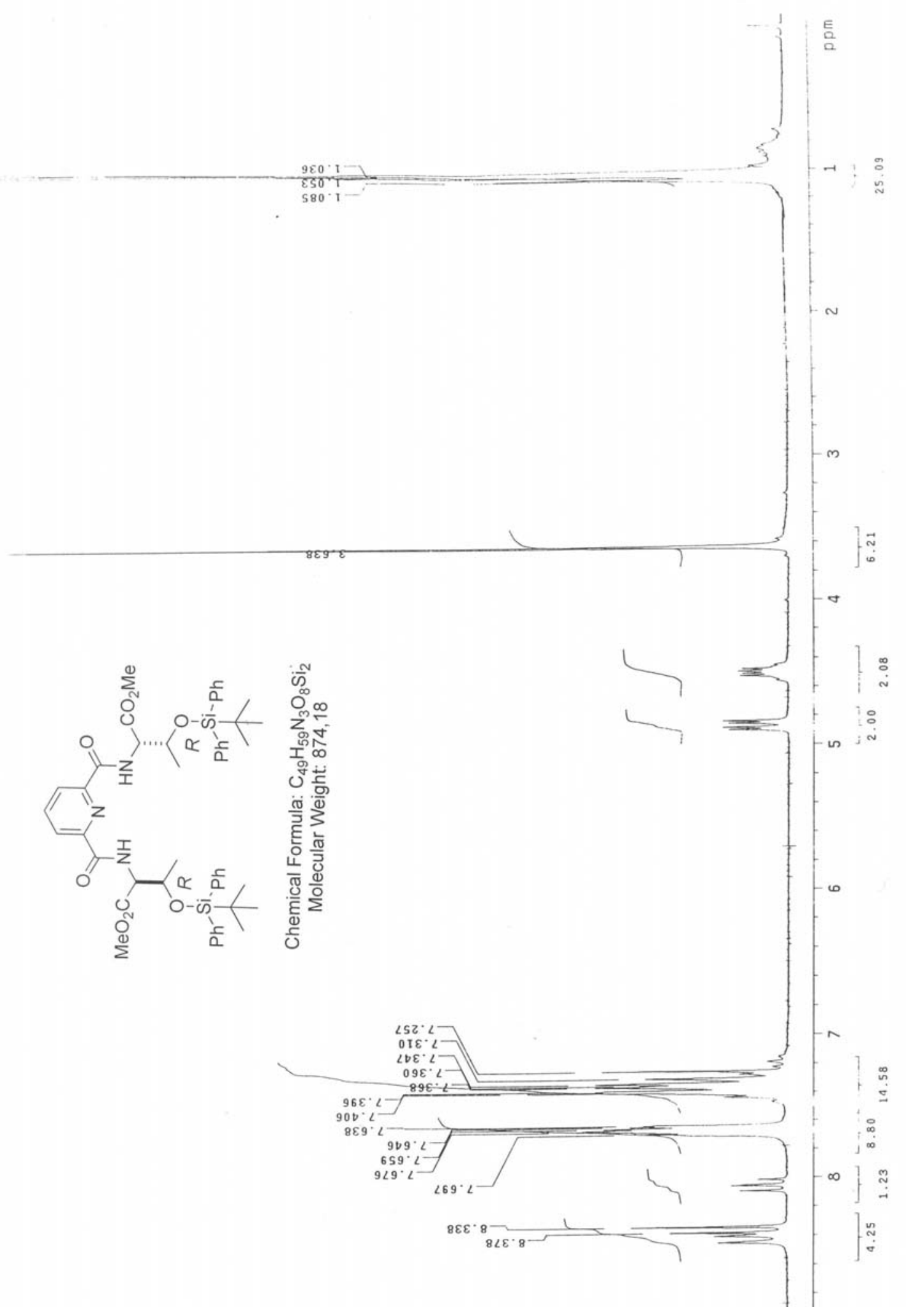


$\angle 88^{\circ} 0 \angle$

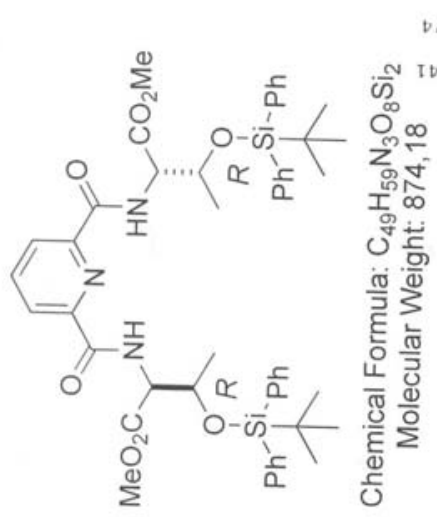

$$
\begin{aligned}
0<98 \\
\angle 00 .
\end{aligned}
$$

200.212
4.24

م.

更我

ปั

3

인

ठ

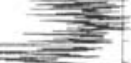

$905^{\circ}$ S2I-
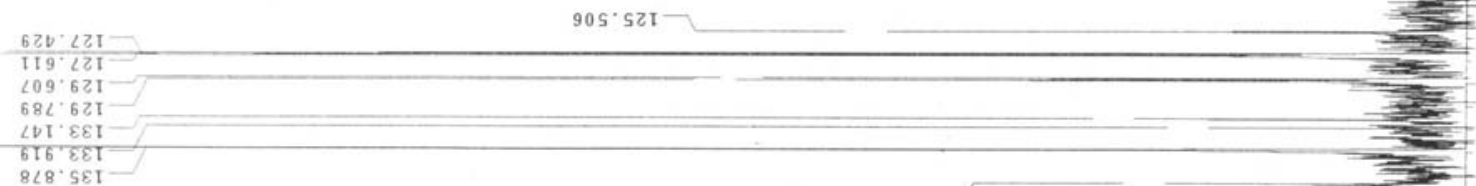
616.88

$\angle 98 \cdot 8 E I-$

SI $6.8 \mathrm{II}^{-}$

$959.89 \mathrm{I}$

$682^{\circ} 0 \angle \mathrm{I}^{-}$ 


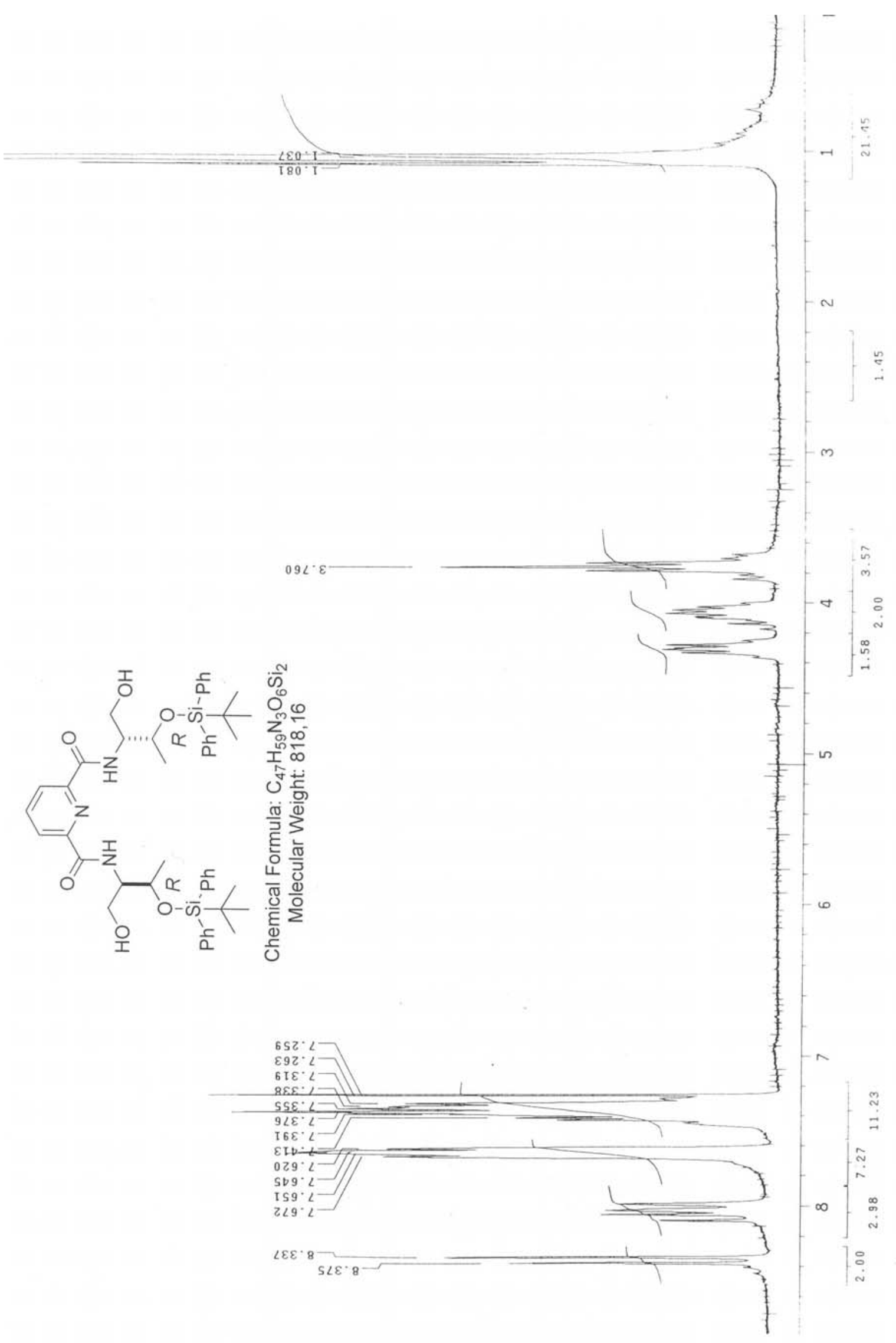




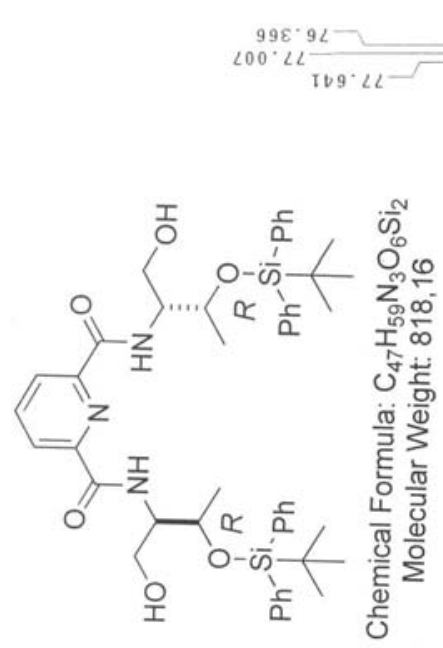

$S \angle 5^{\circ} \angle 2$
$808^{\circ} \angle 2$

I $98^{\circ} \mathrm{SZt}$

$28 L^{\circ} \cdot 62$

$986^{\circ} 6 Z \mathrm{I}$
$586^{\circ} \mathrm{ZEI}$

SSE. EEI
BT L S SE

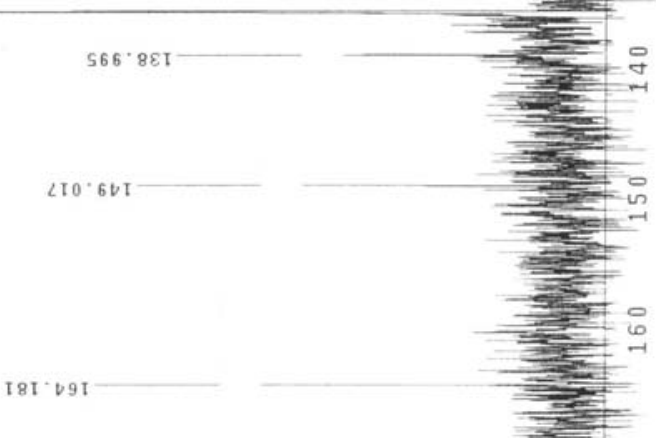




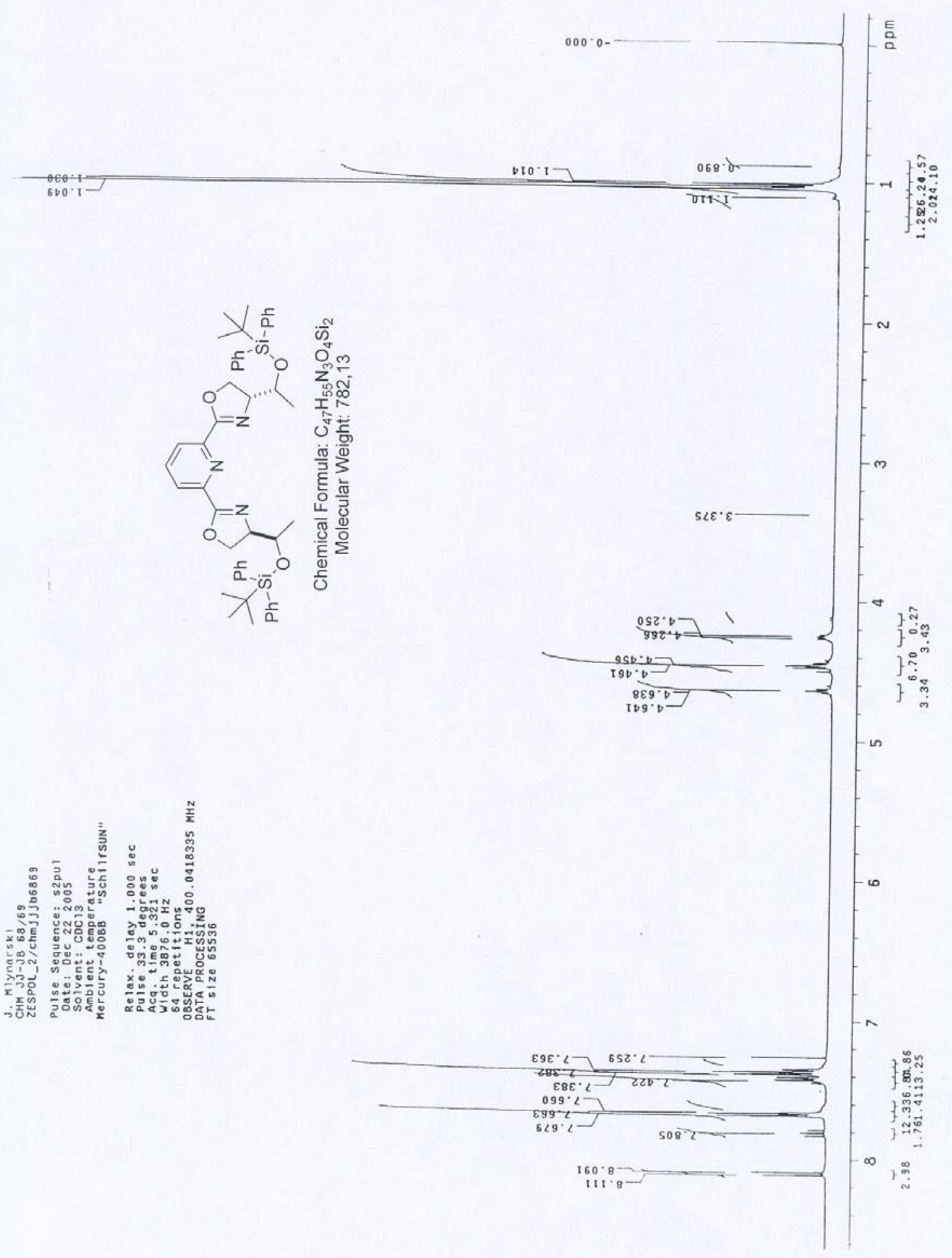




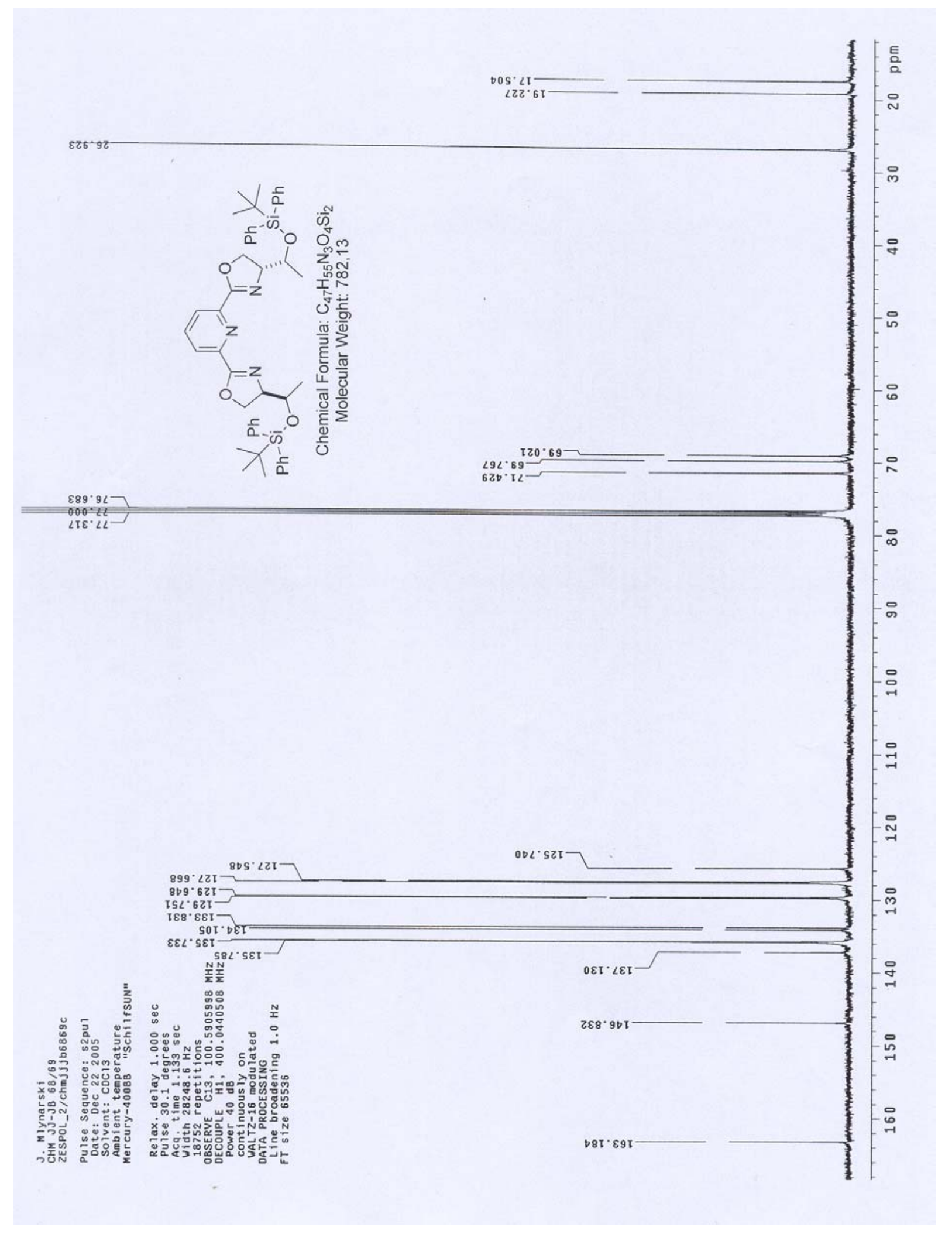


Raport wydrukowano: 03-07-2006 12:04

| jb59a.chr |

Nazwa analizy:

Data utworzenia:

Analiza:

jj-jb-059a

chiralpak adh

Heksan-iPrOH 9:1

$1 \mathrm{ml} / \mathrm{min}$

\begin{tabular}{rrr} 
NUMER & CZAS RETENCJI & \multicolumn{1}{c}{ UDZIAK } \\
PIKU & [min] & PROCENTOWY \\
A/1 & 10,462 & 7,435 \\
A/2 & 13,073 & 87,617 \\
A/3 & 15,695 & 0,837 \\
A/4 & 17,170 & 4,111 \\
SUMA & &
\end{tabular}

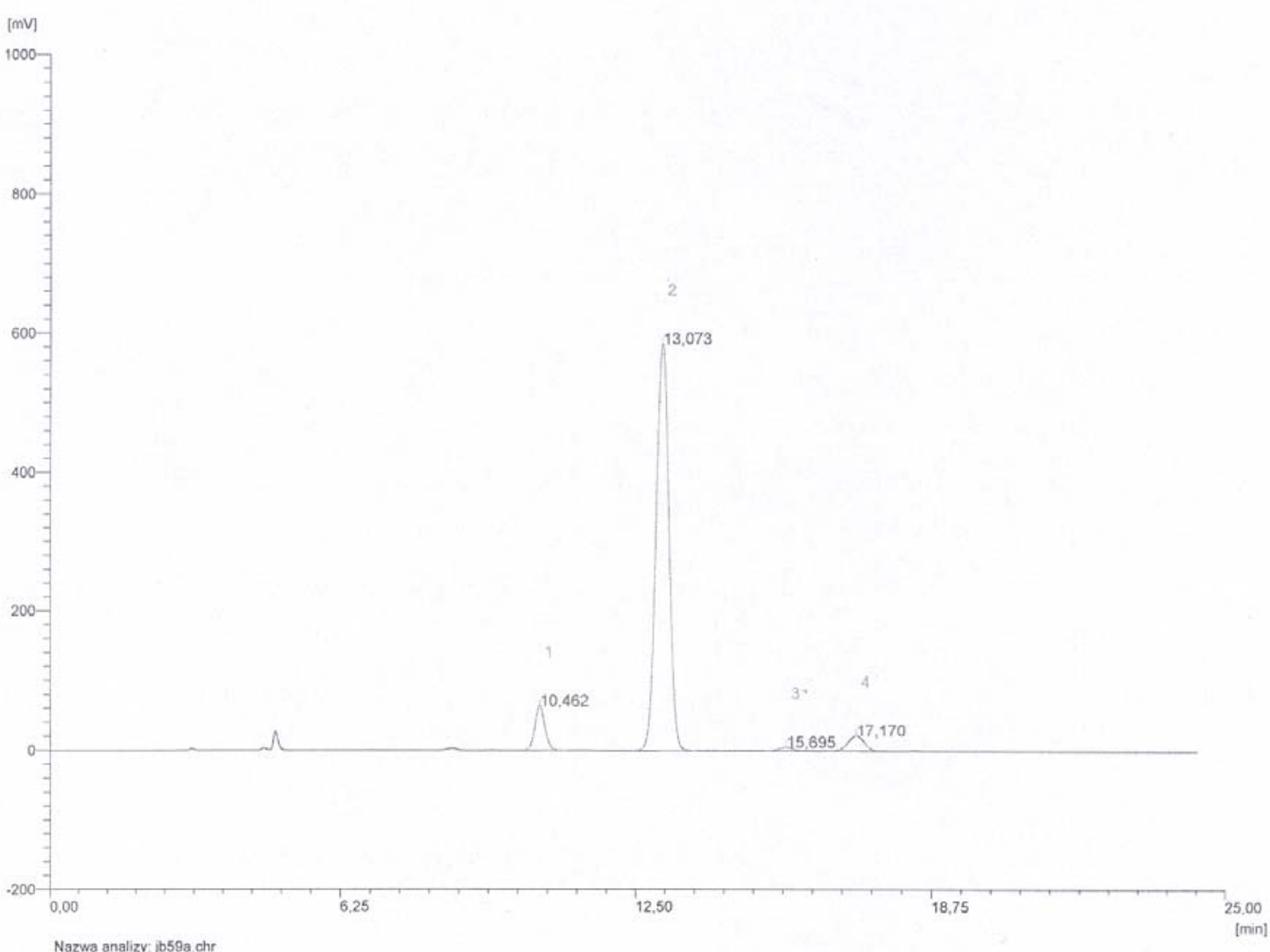

jb59a.chr / gc1.met 01-12-05 11:58 /

20

ChromaX wersja1.2c

UDZIAK
PROCENTOWY
7,435
87,617
0,837
4,111

Chemical Formula: $\mathrm{C}_{16} \mathrm{H}_{16} \mathrm{O}_{2}$ Molecular Weight: 240,3 
Raport wydrukowano: 03-07-2006 12:11 | jb70a.chr |

Nazwa analizy:

jb70a.chr / gc1.met

Data utworzenia:

28-12-05 11:54 /

Analiza:

45

ChromaX wersja1.2c

jj-jb-070a

chiralpak adh

Heksan-iPrOH 9:1

$1 \mathrm{ml} / \mathrm{min}$

\begin{tabular}{rrr} 
NUMER & CZAS RETENCJI & \multicolumn{1}{c}{ UDZIAK } \\
PIKU & {$[\mathrm{min}]$} & PROCENTOWY \\
A/1 & 16,435 & 5,438 \\
A/2 & 18,537 & 88,169 \\
A/3 & 25,885 & 5,283 \\
A/4 & 26,790 & 1,110 \\
SUMA & &
\end{tabular}

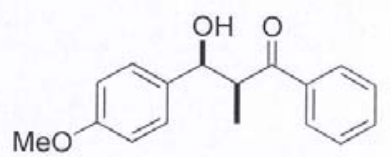

Chemical Formula: $\mathrm{C}_{17} \mathrm{H}_{18} \mathrm{O}_{3}$ Molecular Weight: 270,32

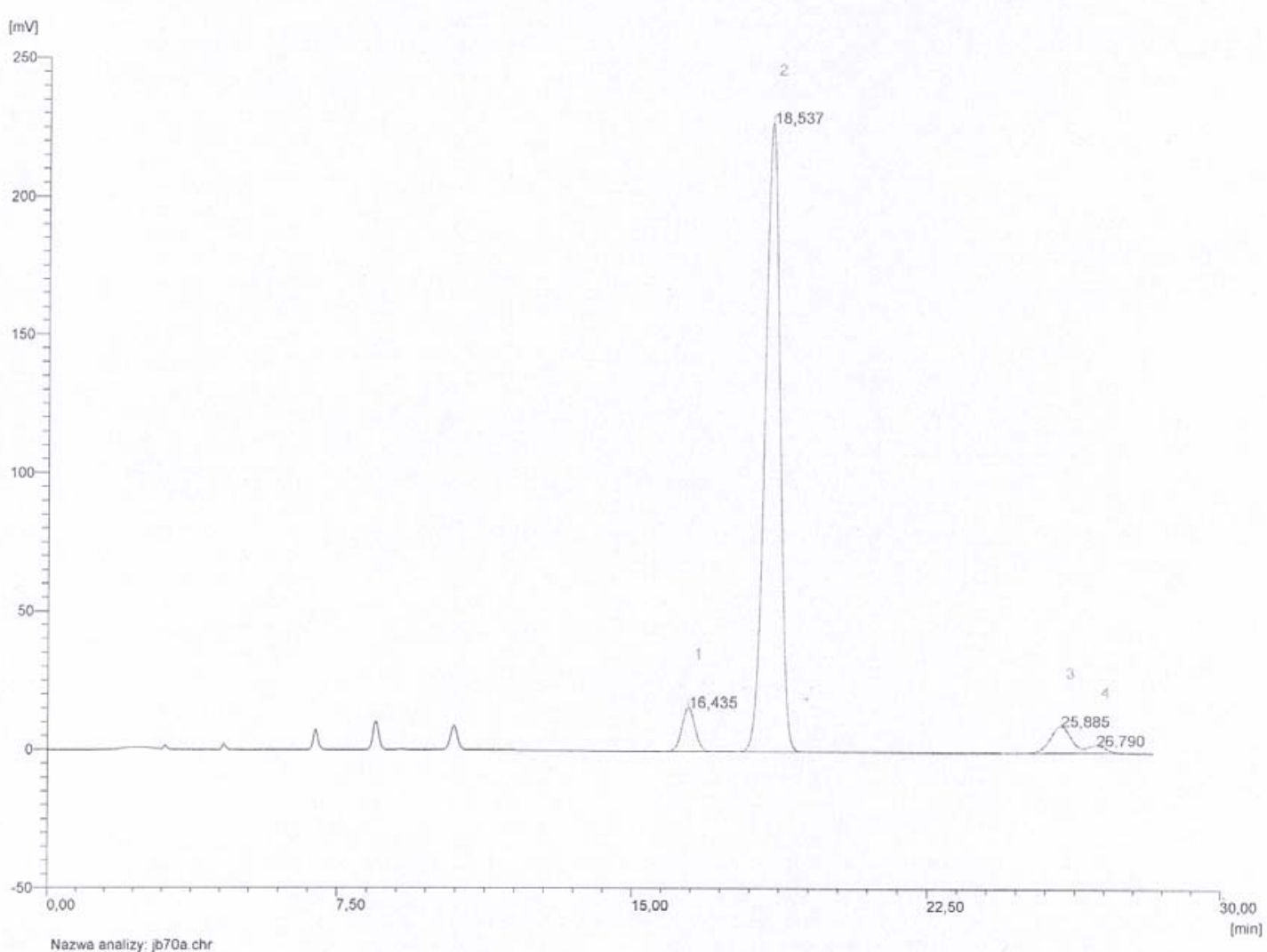


Raport wydrukowano: 03-07-2006 12:13

| jb70b.chr |

Nazwa analizy:

Data utworzenia:

jb70b.chr / gc1.met

Analiza:

28-12-05 13:10

ji-jb-070b

chiralpak adh

Heksan-iPrOH 9:1

$1 \mathrm{ml} / \mathrm{min}$

\begin{tabular}{rrr} 
NUMER & CZAS RETENCJI & \multicolumn{1}{c}{ UDZIAK } \\
PIKU & [min] & PROCENTOWY \\
A/1 & 12,537 & 10,745 \\
A/2 & 19,077 & 82,008 \\
A/3 & 23,575 & 7,248 \\
SUMA & &
\end{tabular}

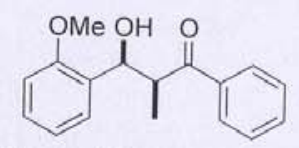

Chemical Formula: $\mathrm{C}_{17} \mathrm{H}_{18} \mathrm{O}_{3}$ Molecular Weight: 270,32

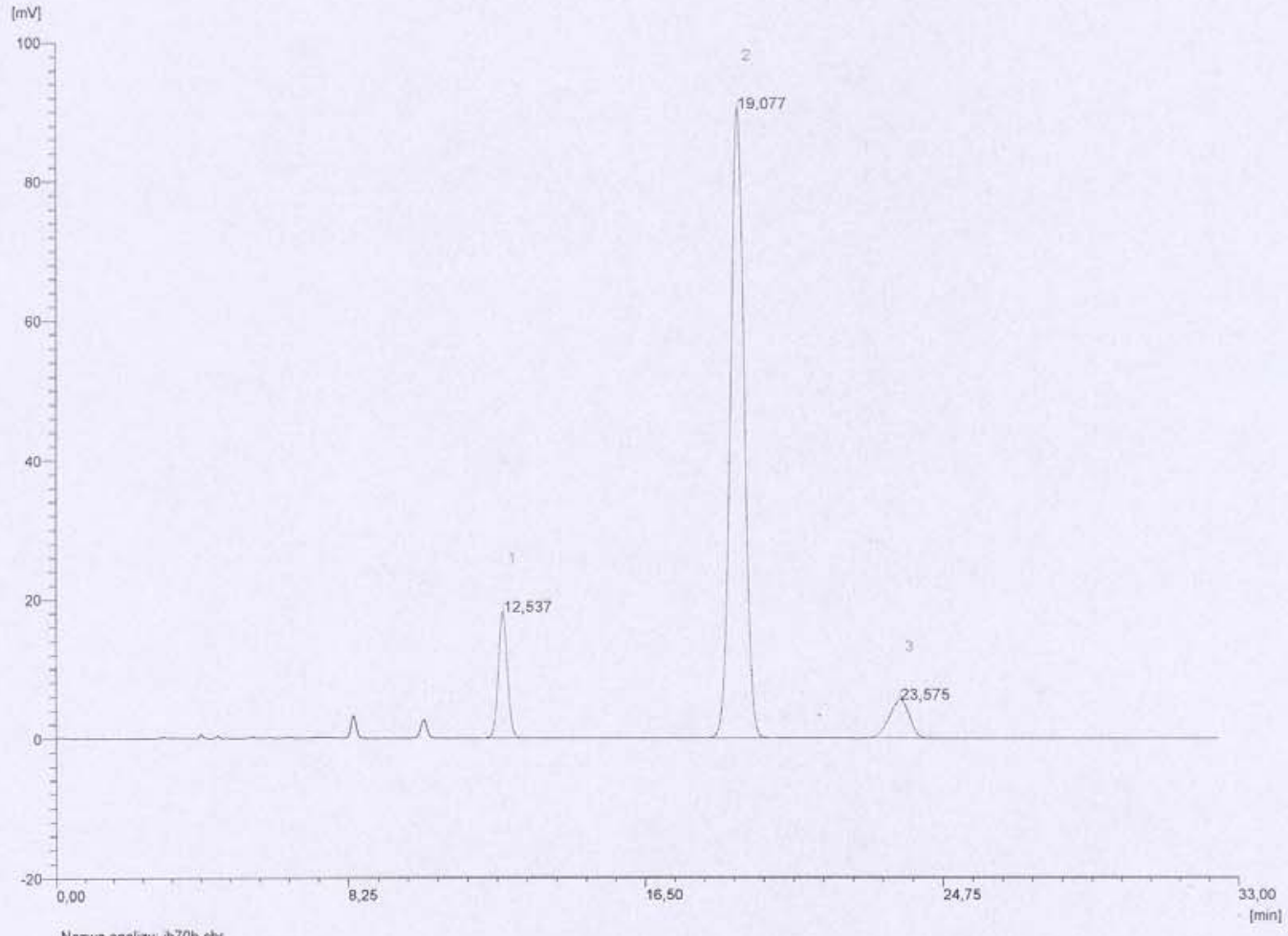


Raport wydrukowano: 03-07-2006 12:15

Nazwa analizy:

Data utworzenia:

Analiza:

jj-jb-073a

chiralpak adh

Heksan-iPrOH 9:1

$1 \mathrm{ml} / \mathrm{min}$

\begin{tabular}{rrr} 
NUMER & CZAS RETENCJI & \multicolumn{1}{c}{ UDZIA } \\
PIKU & [min] & PROCENTOWY \\
A/1 & 10,657 & 10,055 \\
A/2 & 12,085 & 84,675 \\
A/3 & 16,618 & 4,210 \\
A/4 & 17,590 & 1,061 \\
SUMA & &
\end{tabular}

jb73a.chr / gc1.met

03-01-06 14:31 /

22

ChromaX wersja1.2c

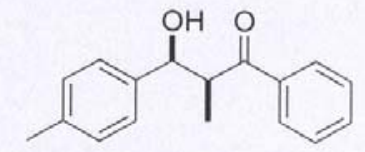

Chemical Formula: $\mathrm{C}_{17} \mathrm{H}_{18} \mathrm{O}_{2}$

Molecular Weight: 254,32

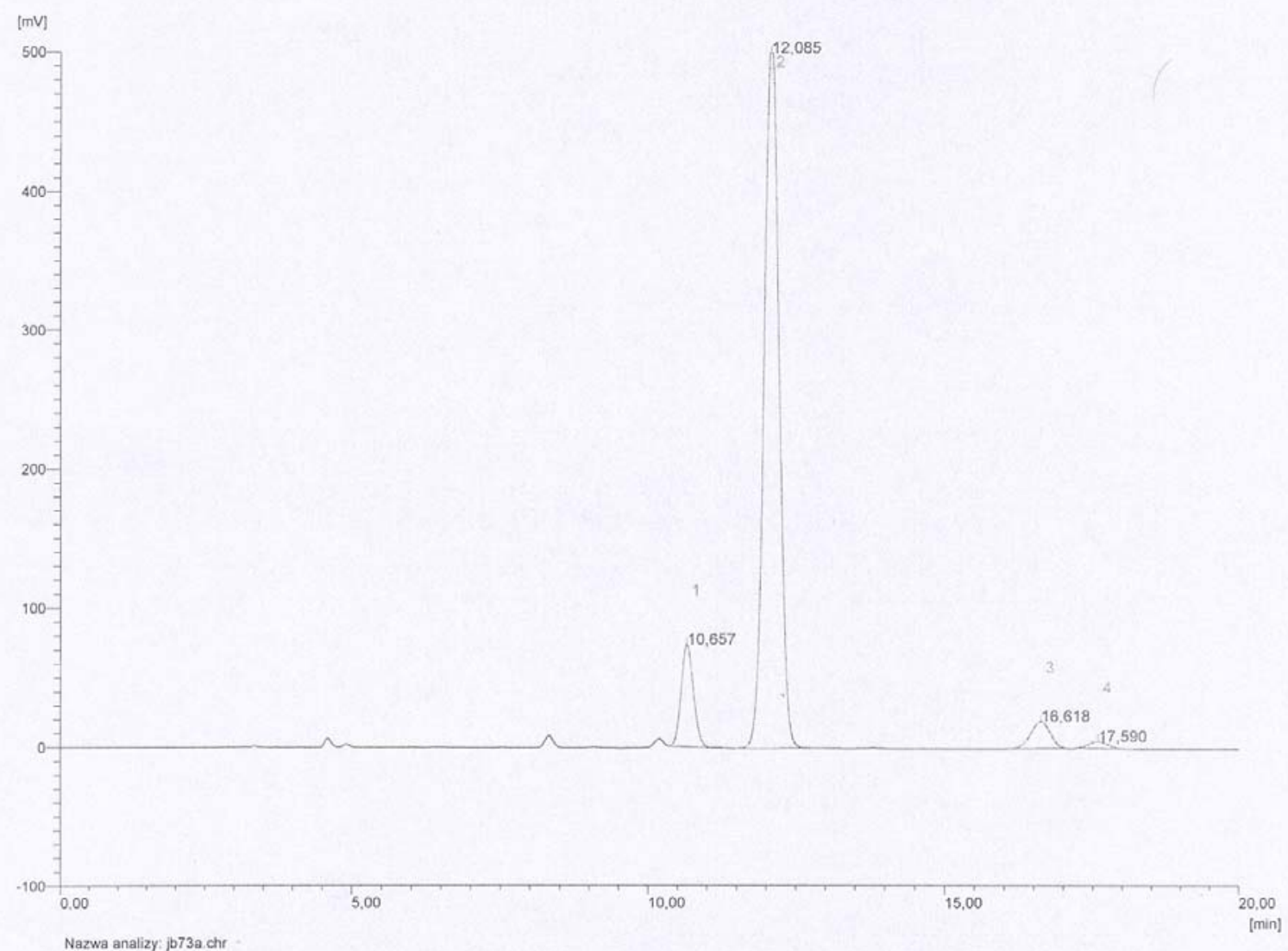


Raport wydrukowano: 03-07-2006 12:20

| jb63b.chr |

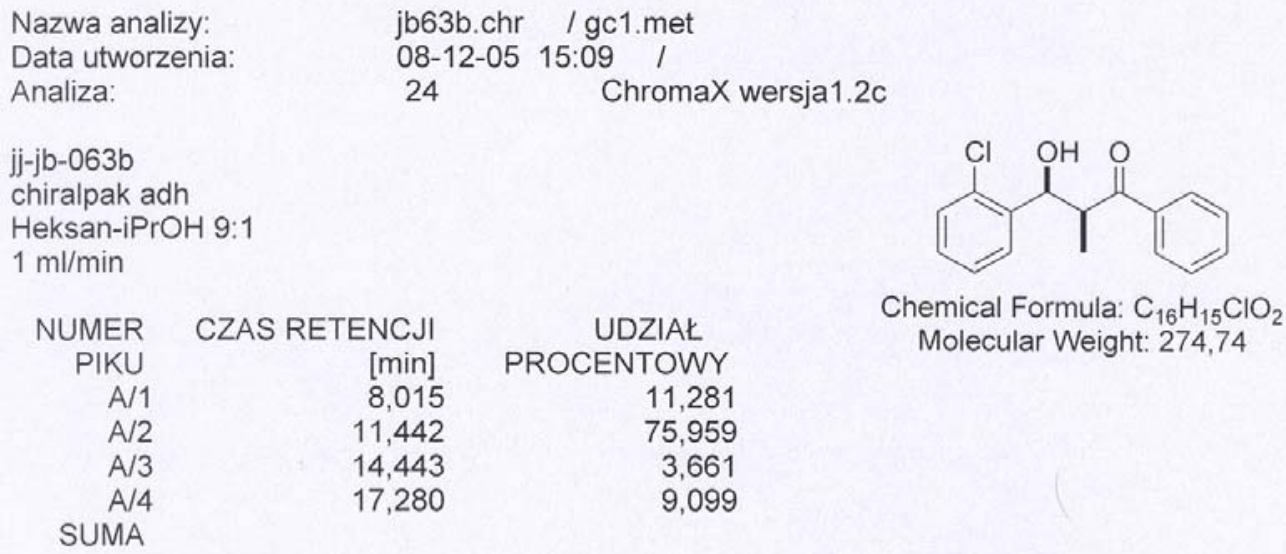

Chemical Formula: $\mathrm{C}_{16} \mathrm{H}_{15} \mathrm{ClO}_{2}$ Molecular Weight: 274,74

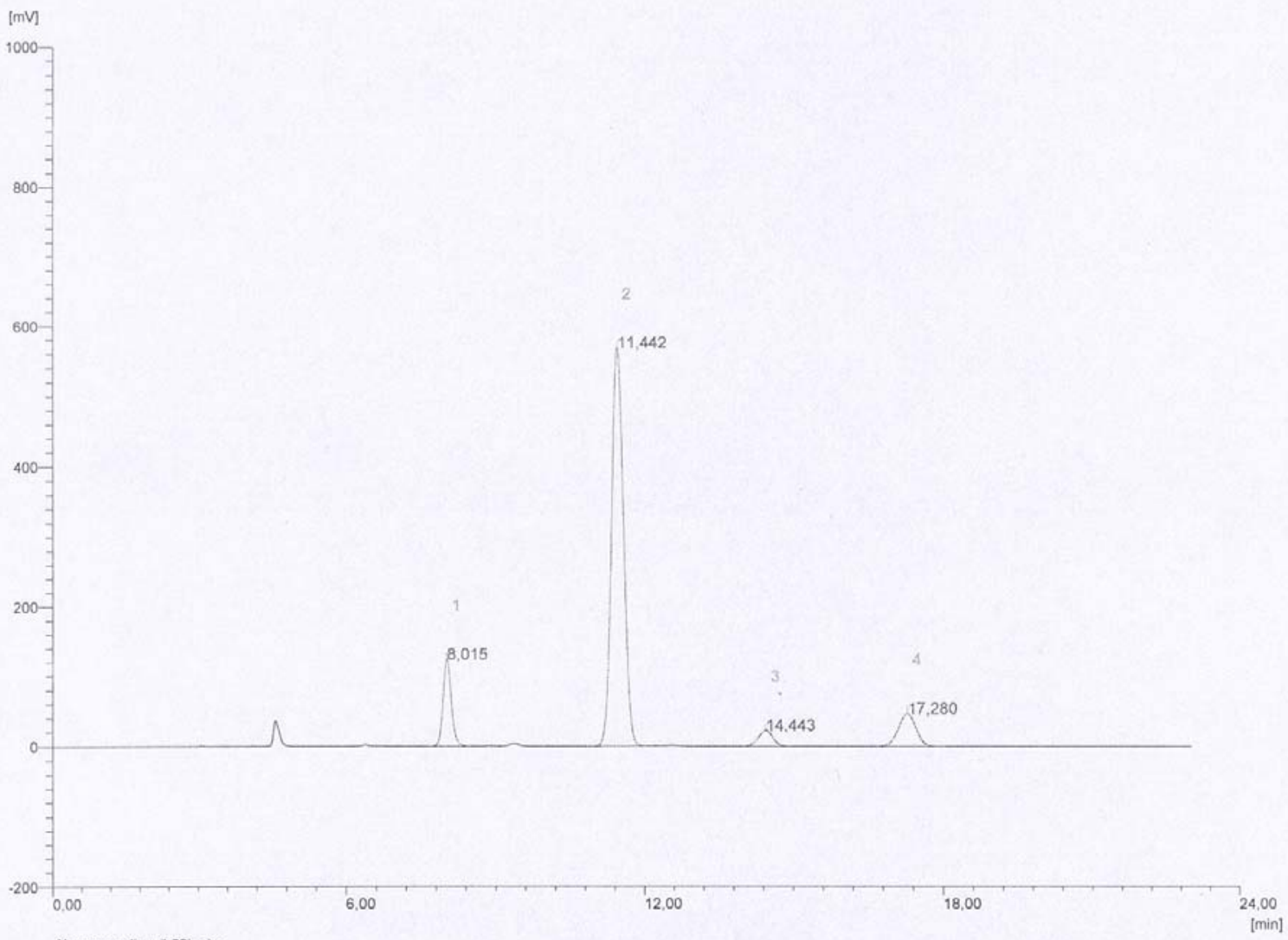

Nazwa analizy: J663b.chs 
Raport wydrukowano: 03-07-2006 12:18

| jb63c.chr |
Nazwa analizy:
Data utworzenia:
jb63c.chr / gc1.met
Analiza:
08-12-05 13:42
23
ChromaX wersja1.2c

\section{jj-jb-063c}
chiralpak adh
Heksan-iPrOH 9:1
$1 \mathrm{ml} / \mathrm{min}$

$\begin{array}{rr}\text { NUMER } & \text { CZAS RETENCJI } \\ \text { PIKU } & \text { [min] } \\ \text { A/1 } & 11,110 \\ \text { A/2 } & 12,487 \\ \text { A/3 } & 15,710 \\ \text { A/4 } & 20,500 \\ \text { SUMA } & \end{array}$
UDZIAK
PROCENTOWY
12,175
79,008
7,023
1,794

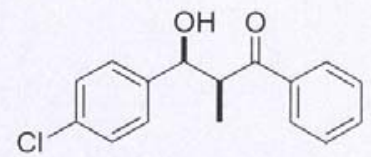
Chemical Formula: $\mathrm{C}_{16} \mathrm{H}_{15} \mathrm{ClO}_{2}$ Molecular Weight: 274,74

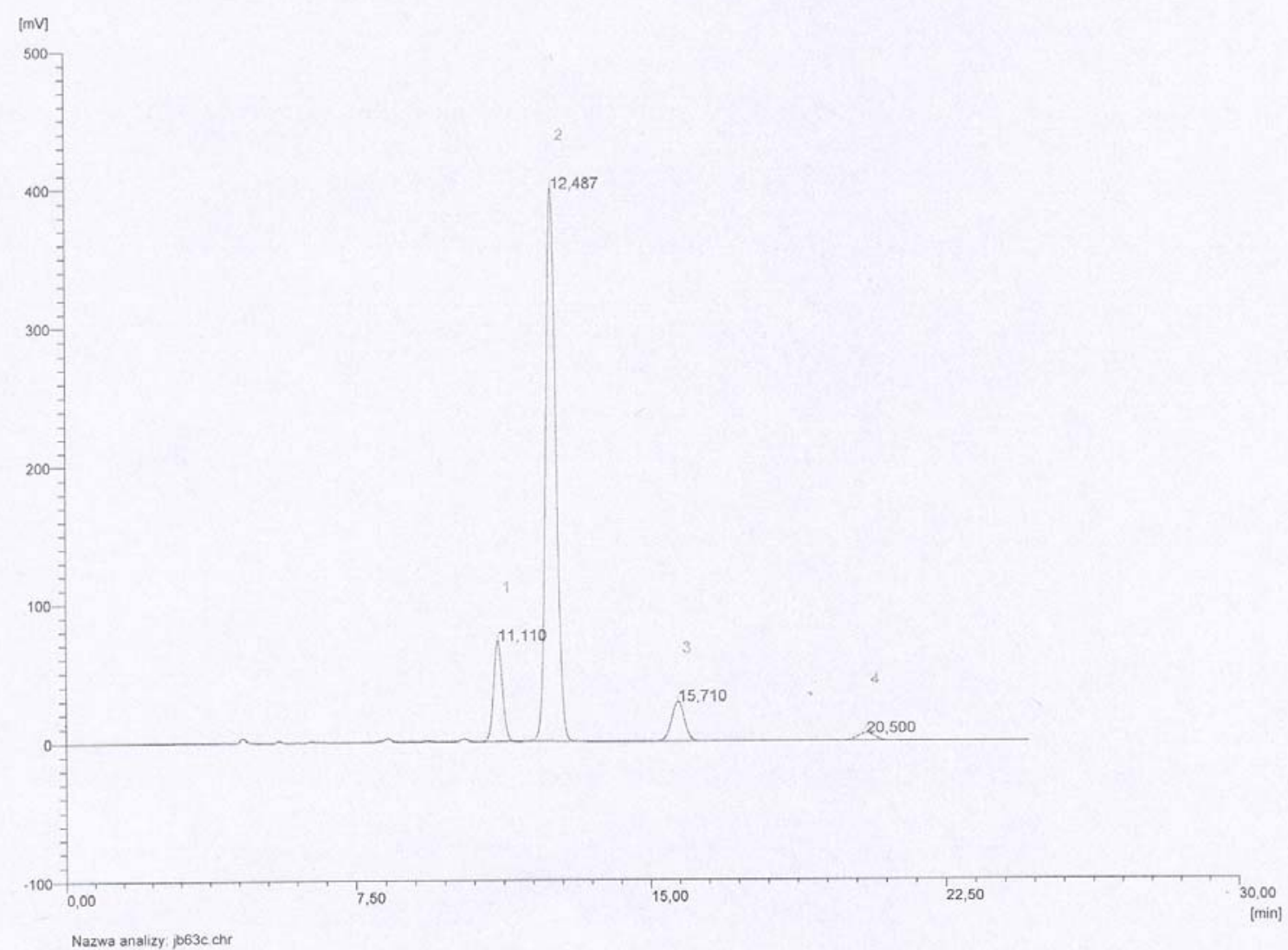


Raport wydrukowano: 03-07-2006 13:18

| jb73b.chr |

Nazwa analizy:

jb73b.chr / gc1.met

Data utworzenia:

Analiza:

27 ChromaX wersja1.2c

jj-jb-073b

chiralpak adh

Heksan-iPrOH 9:1

$1 \mathrm{ml} / \mathrm{min}$

NUMER

CZAS RETENCJI

[min]

19,243

PIKU

$\mathrm{A} / 1$

20,968

PROCENTOWY

$A / 3$

23,315

70,496

$A / 4$

45,308

14,382

SUMA

8,952

6,170

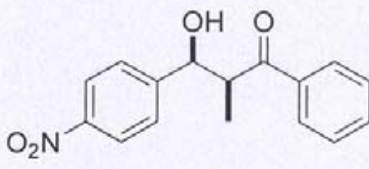

Chemical Formula: $\mathrm{C}_{16} \mathrm{H}_{15} \mathrm{NO}_{4}$ Molecular Weight: 285,29

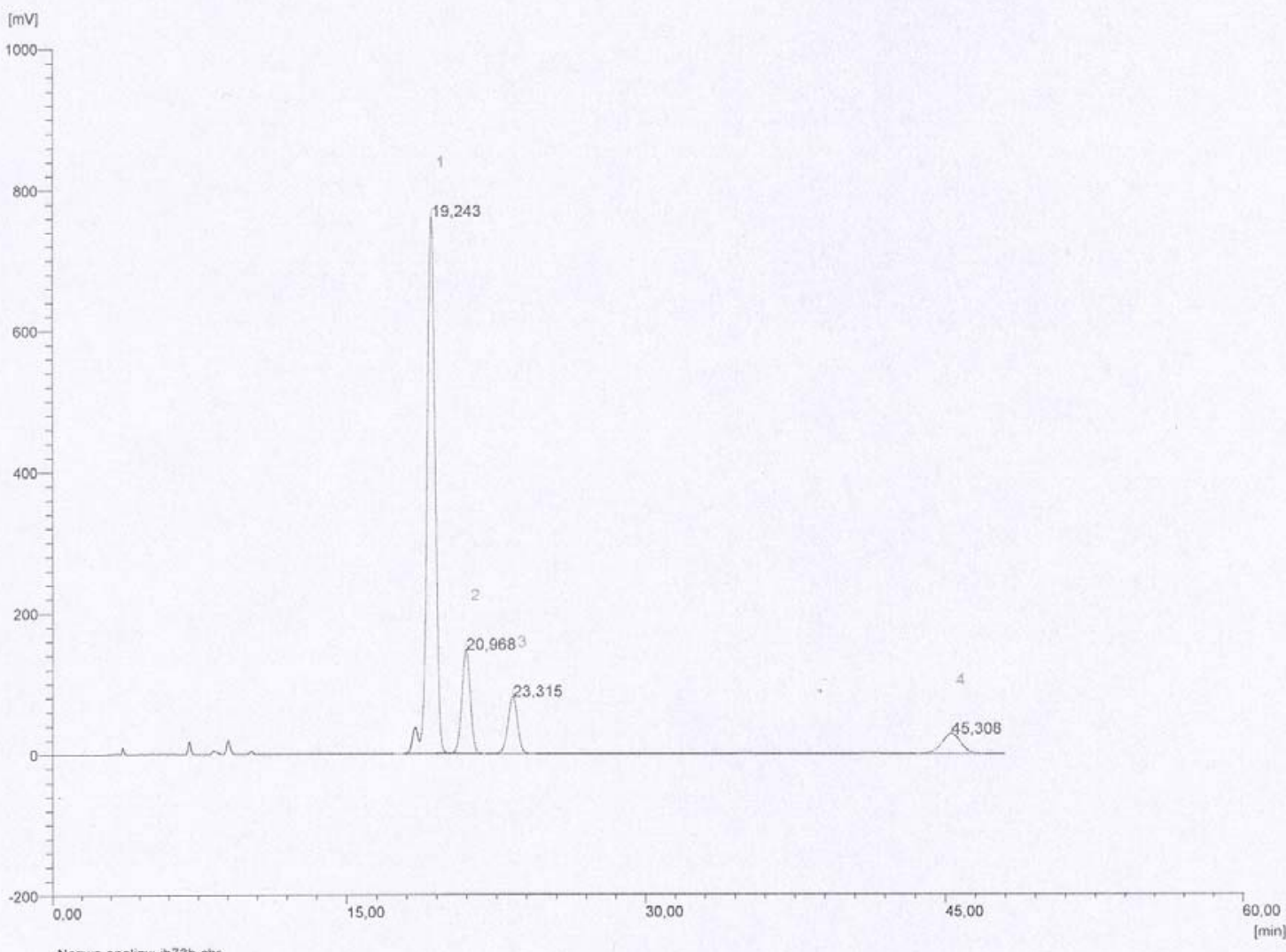


Raport wydrukowano: 03-07-2006 12:22

| jb70c.chr |

Nazwa analizy:

Data utworzenia:

Analiza:

jj-jb-070c

chiralpak adh

Heksan-iPrOH 9:1

$1 \mathrm{ml} / \mathrm{min}$

NUMER

PIKU

$\mathrm{A} / 1$

$\mathrm{A} / 2$

$\mathrm{A} / 3$

$\mathrm{A} / 4$

SUMA jb70c.chr / gc1.met

28-12-05 14:42 ।

25 ChromaX wersja1.2c

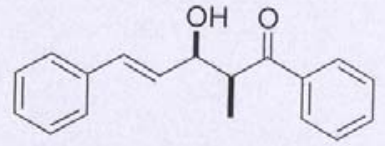

Chemical Formula: $\mathrm{C}_{18} \mathrm{H}_{18} \mathrm{O}_{2}$

Molecular Weight: 266,33

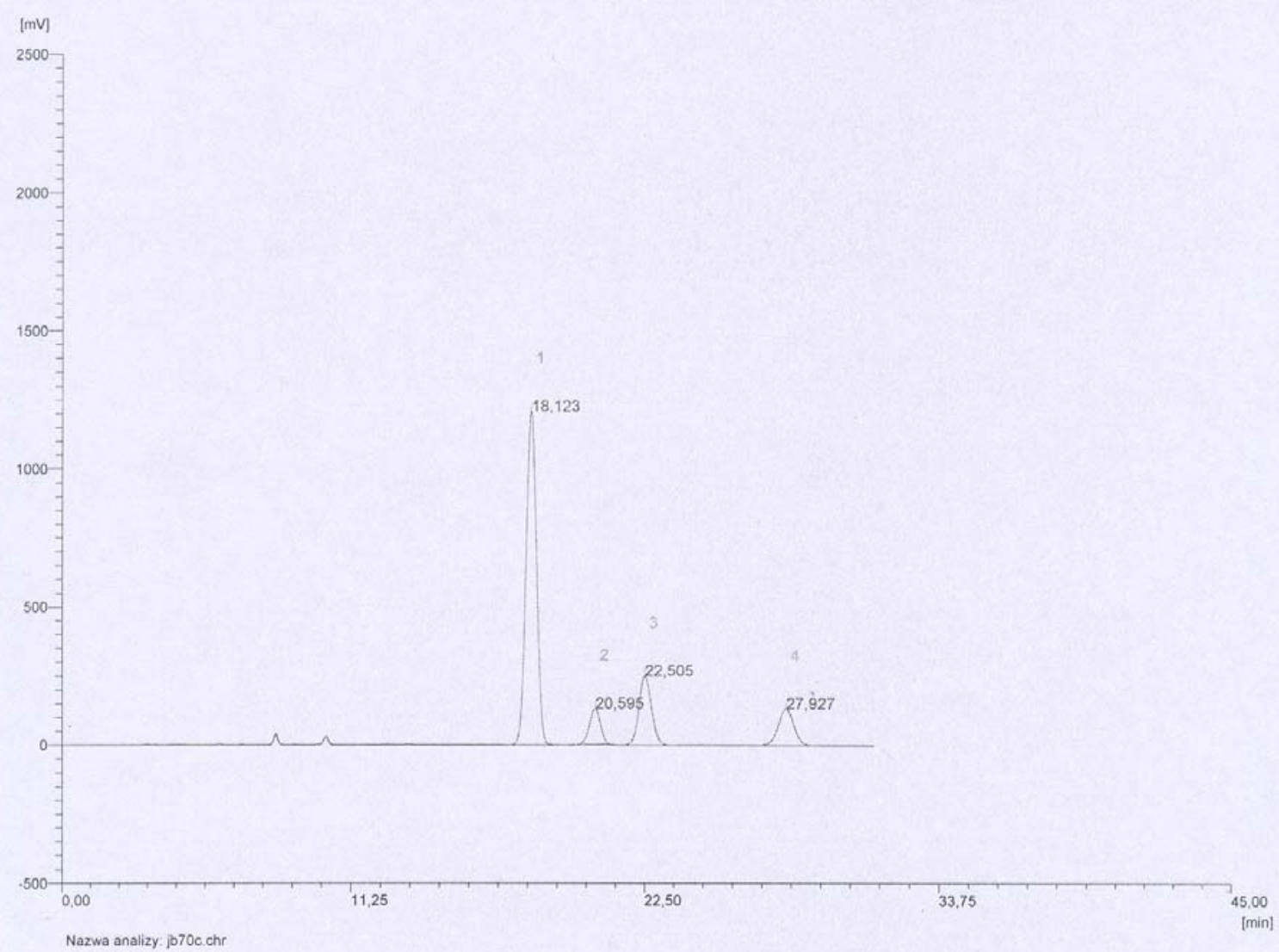

Nazwa analizy: jo $700 \mathrm{ch}$

\section{UDZIAL \\ PROCENTOWY \\ 65,489 \\ 16,279}

[min]

18,123

20,595

22,505

27,927

10,669 
Nazwa analizy:

Data utworzenia:

Analiza:

mly-me-089b

Chiralpak AD-H

Heksan-iPrOH (9:1)

$1 \mathrm{ml} / \mathrm{min}$

\begin{tabular}{rrr} 
NUMER & CZAS RETENCJI & \multicolumn{1}{c}{ UDZIAK } \\
PIKU & [min] & PROCENTOWY \\
A/1 & 16,797 & 82,723 \\
A/2 & 19,033 & 2,583 \\
A/3 & 20,433 & 6,865 \\
A/4 & 25,078 & 7,828 \\
SUMA & &
\end{tabular}

me089b.chr / gc1.met

23-06-06 11:40 /

144 ChromaX wersja1.2c

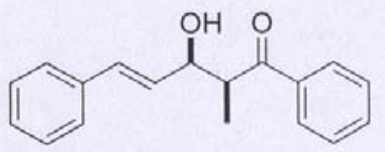

Chemical Formula: $\mathrm{C}_{18} \mathrm{H}_{18} \mathrm{O}_{2}$ Molecular Weight: 266,33

7i, Table 4

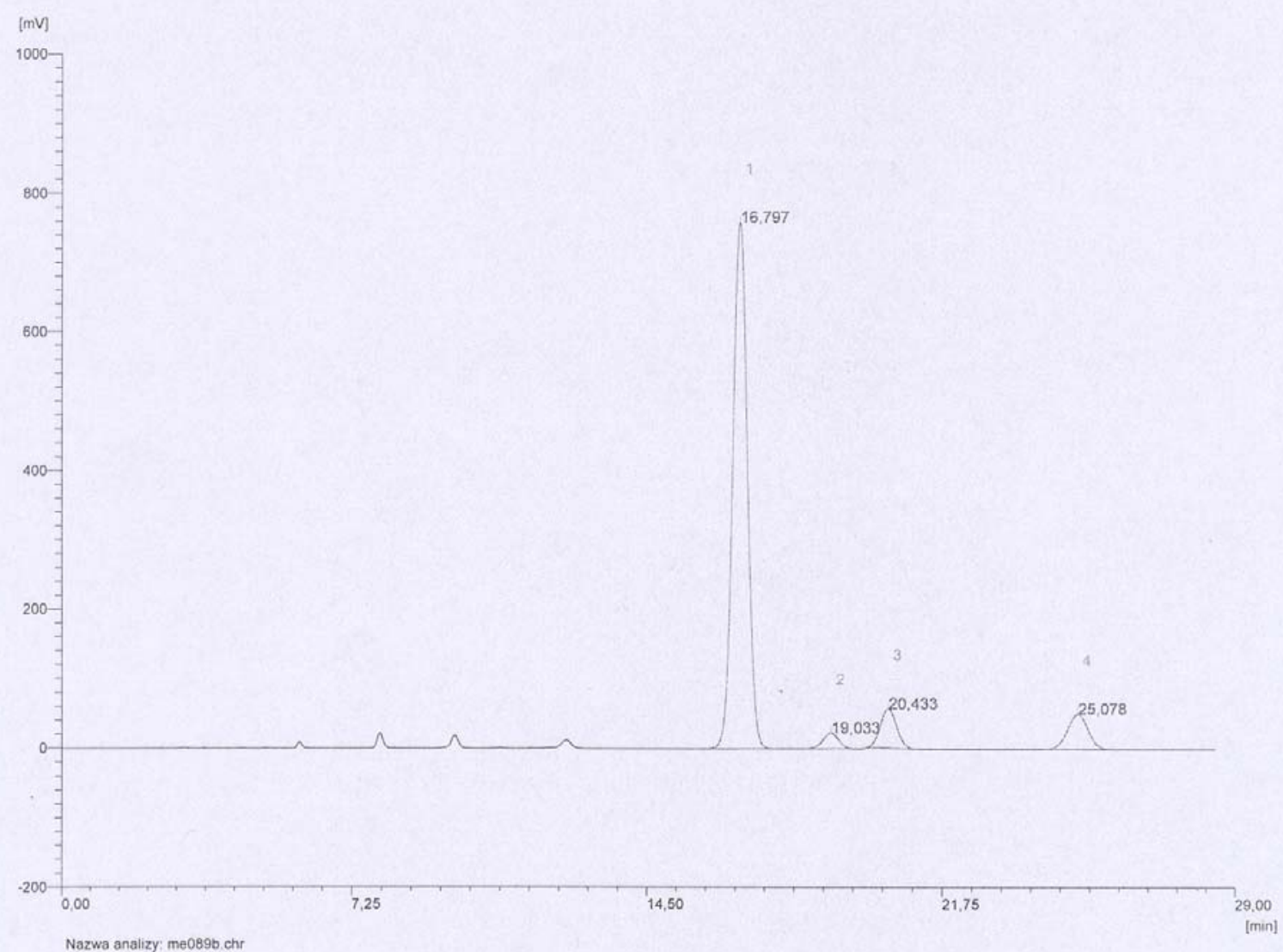


Object name: Time table $-m \_u v(97: 3)$ - Created: $15-02-2005,15: 29$

Path:

\begin{tabular}{|r|r|r|r|r|r|r|}
\hline & \multicolumn{1}{|c|}{$\begin{array}{c}\text { Time } \\
\mathrm{min}\end{array}$} & $\begin{array}{c}\text { Flow } \\
\mathrm{ml} / \mathrm{min}\end{array}$ & $\begin{array}{c}\text { Valve } \\
\% \mathrm{~A}\end{array}$ & $\begin{array}{c}\text { Valve } \\
\% \mathrm{~B}\end{array}$ & D.In & $\begin{array}{c}\text { WLength } \\
1\end{array}$ \\
\hline 1 & 0.00 & 1.000 & 97 & 3 & - & 254 \\
\hline 2 & 0.02 & 1.000 & 97 & 3 & 1 & 254 \\
\hline 3 & 60.00 & 1.000 & 97 & 3 & - & 254 \\
\hline
\end{tabular}

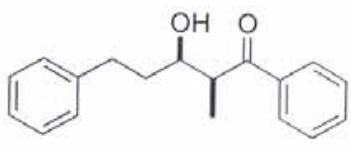

Chemical Formula: $\mathrm{C}_{18} \mathrm{H}_{20} \mathrm{O}_{2}$

Molecular Weight: 268,35

Operator: Joanna

Object name: jb-70d_Det1 - Created: 28-12-2005, 16:26

Path: $>$ Joanna $>$ Pomiary

\begin{tabular}{|c|c|c|c|c|c|c|c|}
\hline & $\begin{array}{c}\text { Ret.time } \\
\text { [min] }\end{array}$ & $\begin{array}{l}\text { Start } \\
\text { [min] }\end{array}$ & $\begin{array}{l}\text { End } \\
\text { [min] }\end{array}$ & $\begin{array}{c}\text { Area } \\
\text { [mAU"min] }\end{array}$ & $\begin{array}{l}\text { Height } \\
\text { [mAU] }\end{array}$ & $\%$ Area & $\begin{array}{l}\text { Width } \\
\text { [min] }\end{array}$ \\
\hline 1 & 11.685 & 11.28 & 12.43 & 183.274 & 489.332 & 16.8352 & 0.356 \\
\hline 2 & 13.069 & 12.58 & 13.95 & 143.304 & 400.995 & 13.1637 & 0.336 \\
\hline 3 & 18.187 & 12.73 & 20.58 & 731.188 & 864.99 & 67.1656 & 0.816 \\
\hline 4 & 28.589 & 27.74 & 29.81 & 30.868 & 39.4736 & 2.8355 & 0.738 \\
\hline
\end{tabular}

Operator: Joanna

Object name: jb-70d_Det1 - Created: 28-12-2005, 15:50

Path: > Joanna $>$ Pomiary

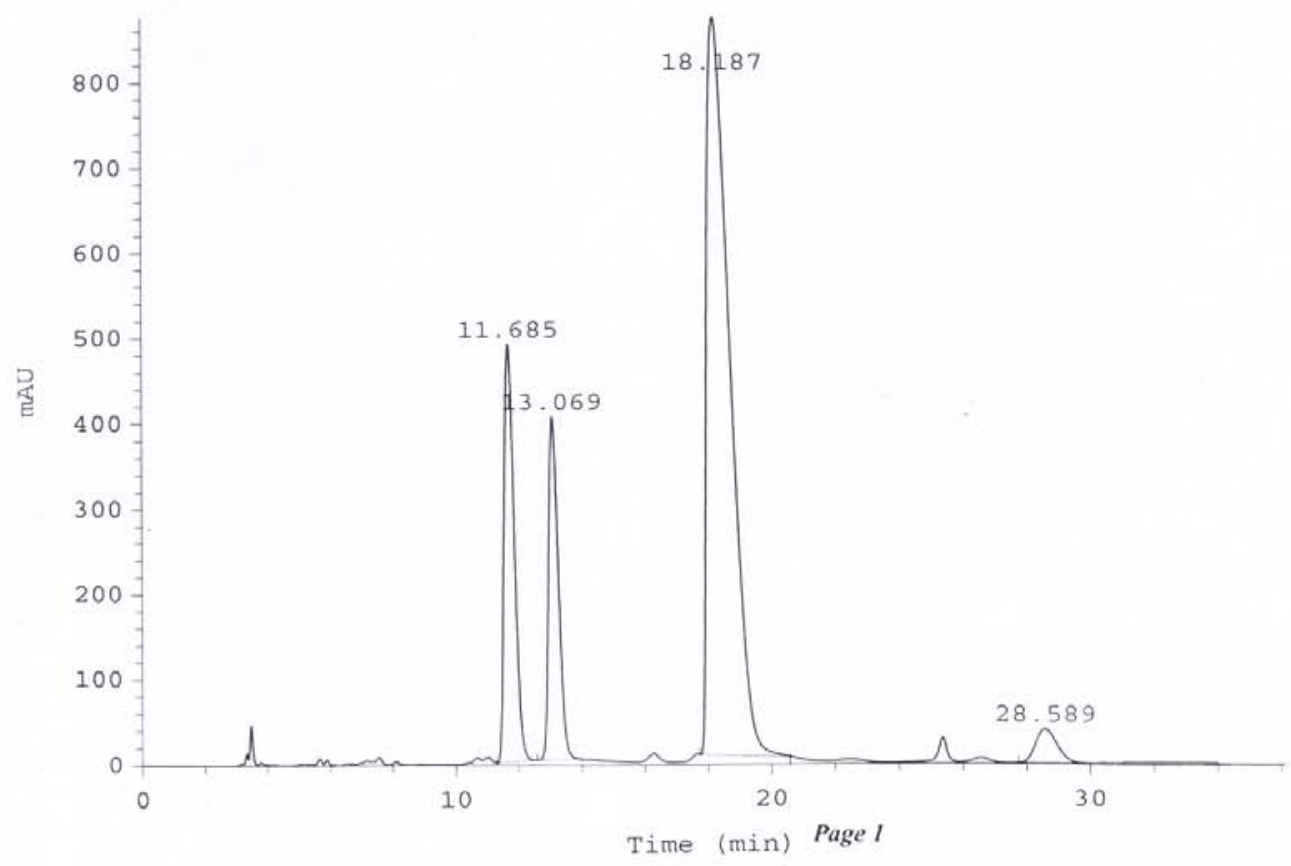


Object name: Time table $-m_{-} \mu v(97: 3)$ - Created: 04-04-2005, 10:54 Path:

\begin{tabular}{|c|c|c|c|c|c|c|}
\hline & $\begin{array}{l}\text { Time } \\
\min \end{array}$ & $\begin{array}{l}\text { Flow } \\
\mathrm{ml} / \mathrm{min}\end{array}$ & $\begin{array}{c}\text { Valve } \\
\% A\end{array}$ & $\begin{array}{c}\text { Valve } \\
\% B\end{array}$ & D.In & WLength \\
\hline 1 & 0.00 & 1000 & 97 & 3 & - & 254 \\
\hline 2 & 0.02 & 1000 & 97 & 3 & 1 & 254 \\
\hline 3 & 60.00 & 1000 & 97 & 3 & - & 254 \\
\hline
\end{tabular}

Operator: Jacek

Object name: me-089c-2/2_Det - Created: 28-06-2006, 12:52 Path: $>$ Jacek $>$ Pomiary $>A \bar{S} H$

\begin{tabular}{|r|r|r|r|r|r|r|r|}
\hline & $\begin{array}{c}\text { Ret.time } \\
{[\mathrm{min}]}\end{array}$ & \multicolumn{1}{|c|}{$\begin{array}{c}\text { Start } \\
{[\mathrm{min}]}\end{array}$} & \multicolumn{1}{c|}{$\begin{array}{c}\text { End } \\
{[\mathrm{min}]}\end{array}$} & \multicolumn{1}{c|}{$\begin{array}{c}\text { Area } \\
{[\mathrm{mAU} * \mathrm{~min}]}\end{array}$} & \multicolumn{1}{c|}{$\begin{array}{l}\text { Height } \\
{[\mathrm{mAU}]}\end{array}$} & \multicolumn{1}{c|}{$\begin{array}{c}\text { \% Area } \\
{[\mathrm{min}]}\end{array}$} \\
\hline 1 & 10.569 & 10.02 & 11.14 & 27.383 & 71.3308 & 7.1924 & 0.364 \\
\hline 2 & 11.702 & 11.15 & 12.30 & 70.6292 & 216.623 & 18.5514 & 0.303 \\
\hline 3 & 15.870 & 14.67 & 17.85 & 277.862 & 435.606 & 72.9830 & 0.616 \\
\hline 4 & 24.755 & 24.37 & 25.60 & 4.84713 & 12.0804 & 1.2731 & 0.376 \\
\hline
\end{tabular}

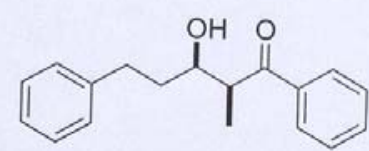

Chemical Formula: $\mathrm{C}_{18} \mathrm{H}_{20} \mathrm{O}_{2}$ Molecular Weight: 268,35

$7 \mathbf{j}$, Table 4

Operator: Jacek

Object name: me-089c-2/2_DetI - Created: 28-06-2006, 12:15

Path: $>$ Jacek $>$ Pomiary $>A \bar{S} H$

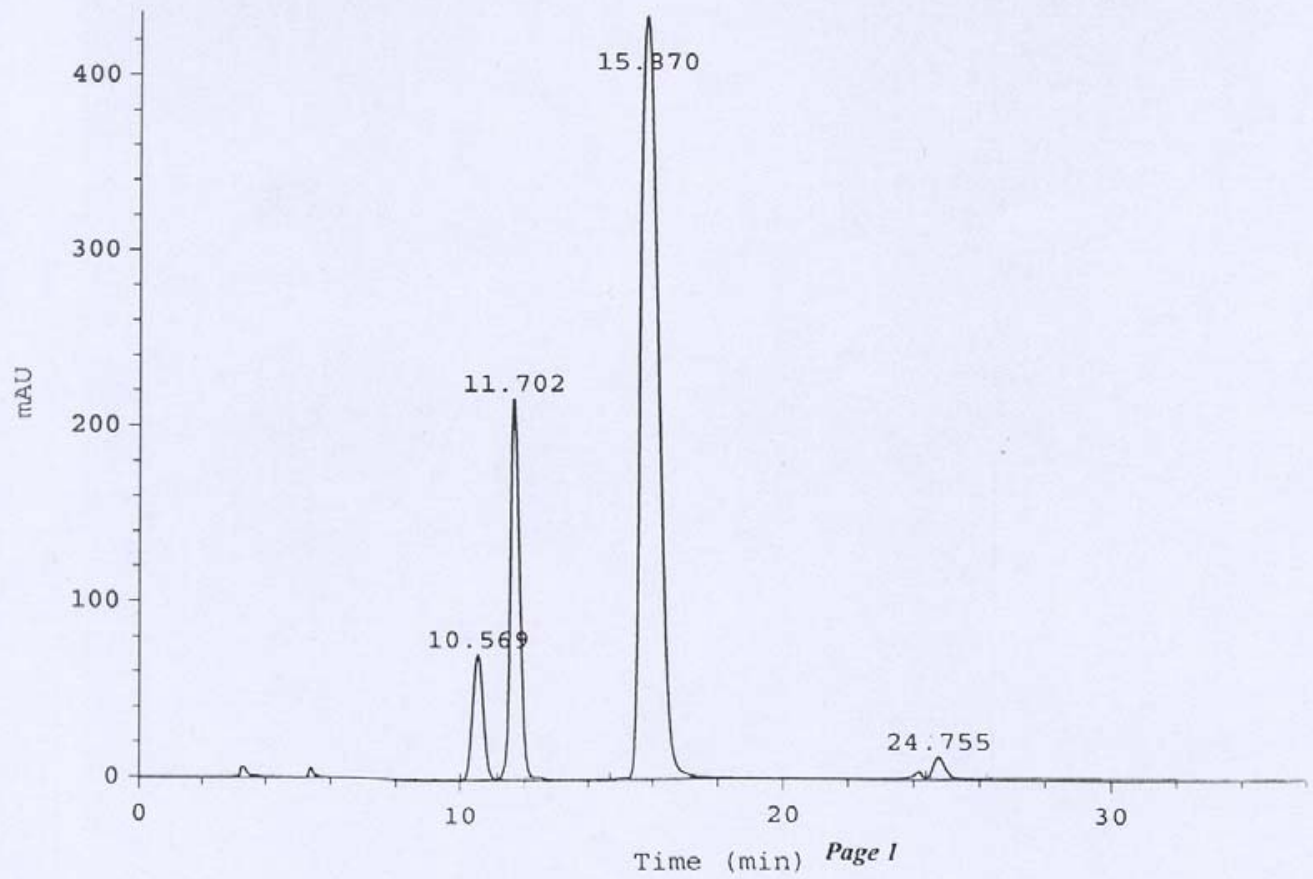


Object name: Time table - $m_{-} u v$ - Created: 17-01-2005, 22:19

Path:

\begin{tabular}{|r|r|r|r|r|r|r|}
\hline & \multicolumn{1}{|c|}{$\begin{array}{c}\text { Time } \\
\text { min }\end{array}$} & \multicolumn{1}{c|}{$\begin{array}{c}\text { Flow } \\
\text { ml/min }\end{array}$} & \multicolumn{1}{|c|}{$\begin{array}{c}\text { Valve } \\
\% A\end{array}$} & $\begin{array}{c}\text { Valve } \\
\% B\end{array}$ & D.In & $\begin{array}{c}\text { WLength } \\
1\end{array}$ \\
\hline 1 & 0.00 & 1.000 & 90 & 10 & - & 254 \\
\hline 2 & 0.02 & 1.000 & 90 & 10 & 1 & 254 \\
\hline 3 & 60.00 & 1.000 & 90 & 10 & - & 254 \\
\hline
\end{tabular}

Operator: Joanno

Object name: jb-76c-(9:1)-ash_DetI -Created: 04-01-2006, 13:39

Path: > Joanna $>$ Pomiary

\begin{tabular}{|c|c|c|c|c|c|c|c|}
\hline & $\begin{array}{c}\text { Ret.time } \\
{[\text { [min] }}\end{array}$ & $\begin{array}{l}\text { Start } \\
{[\mathrm{min}]}\end{array}$ & $\begin{array}{l}\text { End } \\
\text { [min] }\end{array}$ & $\begin{array}{c}\text { Area } \\
{\left[m A U^{*} \min \right]}\end{array}$ & $\begin{array}{l}\text { Height } \\
\text { [MAU] }\end{array}$ & $\%$ Area & $\begin{array}{l}\text { Width } \\
\text { [min] }\end{array}$ \\
\hline 1 & 14.154 & 13.69 & 17.02 & 2419.95 & 2565.3 & 76.0756 & 0.918 \\
\hline 2 & 19.773 & 19.21 & 21.36 & 761.031 & 961.123 & 23.9244 & 0.763 \\
\hline
\end{tabular}

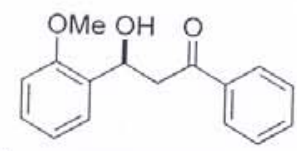

Chemical Formula: $\mathrm{C}_{16} \mathrm{H}_{16} \mathrm{O}_{3}$ Molecular Weight: 256,3

Operator: Joanna

Object name: jb-76c-(9:1)-ash_Det1 - Created: 04-01-2006, 13:11

Path: $>$ Joanna $>$ Pomiary

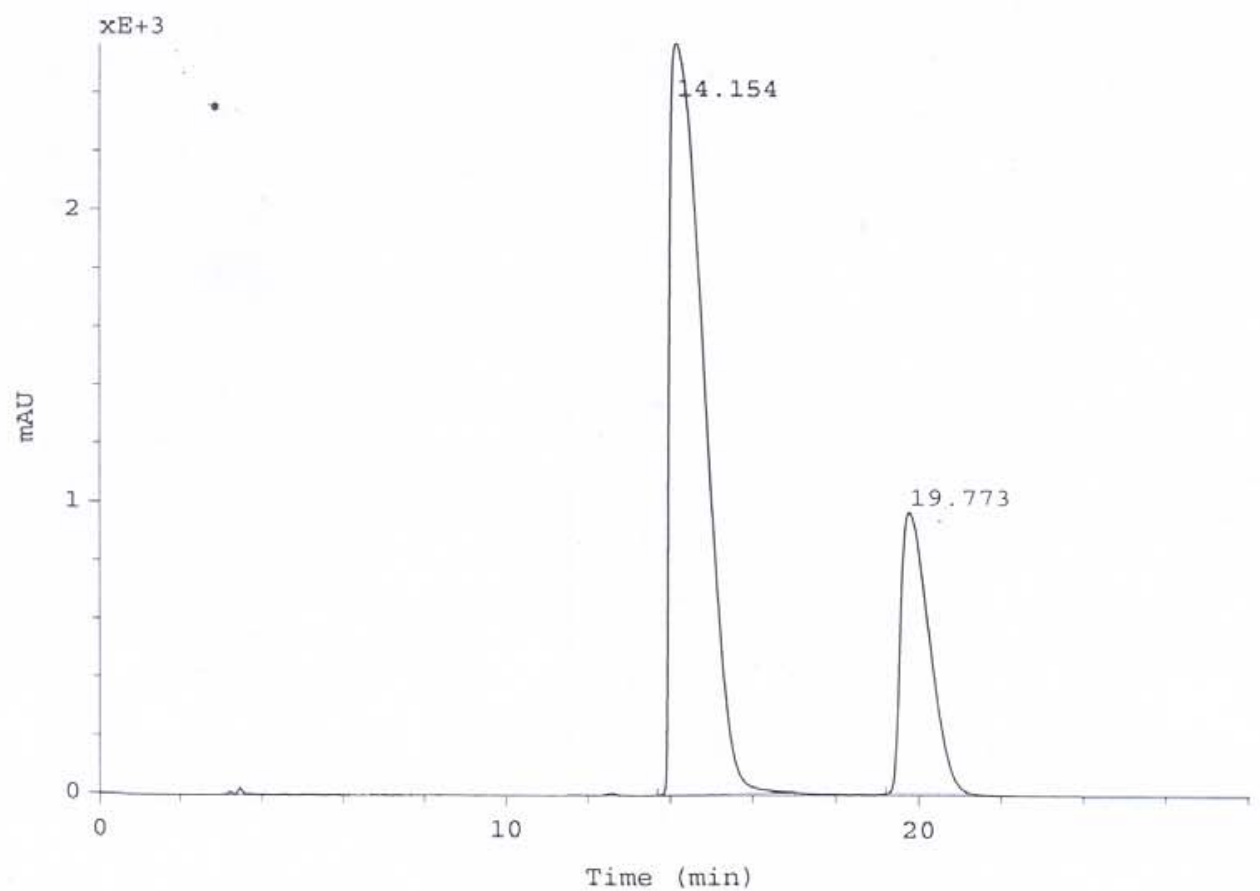

Page I 